\title{
Daniel Sartor
}

Dinâmica temporal e influência de variáveis

ambientais no recrutamento de peixes recifais do Banco dos Abrolhos, Brasil

Temporal dynamics and influence of environmental variables in the recruitment of reef fishes of the Abrolhos Bank, Brazil

São Paulo 


\section{Daniel Sartor}

UNIVERSIDADE DE SÃO PAULO

Dinâmica temporal e influência de variáveis ambientais no recrutamento de peixes recifais do Banco dos Abrolhos, Brasil

Temporal dynamics and influence of environmental variables in the recruitment of reef fishes of the Abrolhos Bank, Brazil

VERSÃO CORRIGIDA

Dissertação apresentada ao Instituto de Biociências da Universidade de São Paulo para obtenção do título de Mestre em Ciências, na Área de Ecologia. Orientador: Prof. Dr. Sérgio Rosso Coorientador: Prof. Dr. Ronaldo Bastos Francini Filho

São Paulo 


\section{Ficha catalográfica}

Sartor, Daniel.

Dinâmica temporal e influência de variáveis ambientais no recrutamento de peixes recifais do Banco dos Abrolhos, Brasil / Daniel Sartor; orientador Sergio Rosso. -- São Paulo, 2015.

\section{$50 \mathrm{f}$.}

Dissertação (Mestrado) - Instituto de Biociências da Universidade de São Paulo. Departamento de Ecologia.

1. Área marinha protegida. 2. Recruta. 3. Recife de coral. 4. Área de berçário. 5. Boosted Regression Trees I. Sérgio Rosso. II. Universidade de São Paulo. Instituto de Biociências. Departamento de Departamento de Fisiologia Geral. III. Título.

\section{Comissão Julgadora:}




\section{AGRADECIMENTOS}

Agradeço a todos que contribuíram diretamente para meu crescimento como biólogo e cientista, tanto com discussões sobre trabalhos, coletas em campo ou conselhos acadêmicos. São tantas pessoas que fica até difícil de lembrar: Matheus Oliveira Freitas, Guilherme Henrique Pereira Filho, Fernando Zaniolo Gibran, Rodrigo Leão de Moura, Aline Alves, Fernando Coreixas de Moraes, Zá, Maurício, Carlinhos, Todinho além de tantos outros que em algum momento auxiliaram na coleta e análise de dados do monitoramento de Abrolhos.

Também sou grato a todos que estiveram envolvidos nos projetos de conservação de Abrolhos que forneceram aporte financeiro e logístico para a pesquisa: projeto Rede Abrolhos (Edital MCT/CNPq/MEC/CAPES/FNDCT Ação Transversal/FAPs № 47/2010 - SISBIOTA), PELD Abrolhos (CHAMADA MCTI/CNPq/FAPs № 34/2012), Pró-Abrolhos (Programa Institutos do Milênio CNPq), programa Marine Management Areas Science, ICMBio (Parque Nacional Marinho Abrolhos/ Resex de Corumbaus) e Conservaçao Internacional. Foi sem dúvidas um grande prazer poder mergulhar e ajudar na conservação desse paraíso que é Abrolhos.

Obrigado a todos meus amigos, pelos momentos de descontração. Valeu Robson, Mudinho, CPV, Caio, Figura, Natasha, Marina, Victor, Baloo, Rufus, Zé, Welson, além de tantos outros. Obrigado especialmente à Bitela, minha guia em assuntos da USP, e ao Lumbriga e Tatai pela constante hospedagem durante disciplinas condensadas.

Obrigado Paulo Roberto Guimarães Junior, Ronaldo Bastos FRancini Filho e Sérgio Rosso por todas as ótimas considerações durante as reuniões de 
comitê. Também agradeço a todos os professores e funcionários da USP que me ajudaram durante esses dois anos de Mestrado. Agradeço ao professor Ronaldo Bastos Francini Filho pela constante ajuda em campo e coorientação e ao professor Sérgio, por ter me dado o privilégio de ser seu orientado e ter me ajudado em todas as fases do mestrado. Tenha certeza que esses dois anos me fizeram admirá-lo como pesquisador e como pessoa e que levarei todo esse aprendizado para minha futura vida acadêmica e pessoal.

Agradeço a meu pai, minha mãe e meu irmão por constante apoio e incentivo durante toda minha jornada como biólogo e pessoa. Sem dúvida são eles, mais meus parentes caninos e felinos, a melhor família que alguém pode ter.

Agradeço especialmente a minha linda namorada Isabela Cioffi, a qual amo tanto e que me ajudou no mestrado quando foi preciso, me aguentou quando estive estressado, me colocou pra cima quando desanimei e, resumindo, esteve sempre ao meu lado. Também não posso esquecer de toda a enorme e animada família Cioffi.

Muito obrigado a todos da Captain Dive que contribuíram para minha formação como mergulhador, afinal foi a paixão pelo mergulho que me direcionou para a biologia marinha a quase dez anos atrás.

Resumindo, este trabalho não é só meu, é nosso. 


\section{ÍNDICE}

\section{Lista de figuras}

\section{Lista de tabelas}

\section{Resumo}

\section{Abstract}

1. Introdução

2. Material e métodos 4

2.1. Região de estudo 4

2.2. Delineamento amostral 5

2.3. Padrões espaço-temporais do recrutamento 9

2.4. Resposta do recrutamento a variáveis ambientais 11

$\begin{array}{lr}\text { 3. Resultados } & 14\end{array}$

3.1. Padrões espaço-temporais do recrutamento 14

3.2. Resposta do recrutamento a variáveis ambientais 15

$\begin{array}{lr}\text { 4. Discussão } & 19\end{array}$

4.1. Padrões espaço-temporais do recrutamento 19

4.2. Resposta do recrutamento a variáveis ambientais $\quad 21$

$\begin{array}{ll}\text { 5. Conclusões } & 27\end{array}$

6. Bibliografia 29

7. Anexos 36 


\section{Lista de figuras}

Figura 1: Croquis Topográfico do Banco dos Abrolhos - BA, mostrando suas cinco áreas recifais $A, B, C, D$ e E.

Figura 2: Recrutamento médio no período de 2001 a 2008. Densidade de recrutas (média dos anos) nos diferentes sítios de amostragem para 10 espécies de peixes recifais no Banco dos Abrolhos - BA (dados provenientes de amostragens entre 2001 e 2008).

Figura 3: Recrutamento médio no período de 2006 a 2014: Densidade de recrutas (média dos anos) nos diferentes sítios de amostragem para 12 espécies de peixes recifais nos Abrolhos - BA (dados provenientes de amostragens entre 2006 e 2014).

17

Figura 4: Gráficos de dependência parcial das variáveis ambientais mais influentes (influencia relativa > 10\%) no recrutamento de 10 espécies de peixes recifais de Abrolhos - BA. 


\section{Lista de tabelas}

Tabela 1: Caracterização ambiental dos locais de amostragem e indicação dos locais envolvidos em cada análise.

Tabela 2: Listagem de espécies de peixes recifais consideradas importantes neste estudo com o tamanho dos recrutas, hábito alimentar e inclusão ou não em cada análise.

Tabela 3: Variáveis ambientais utilizadas nas análises BRT: códigos, descrições e unidades de medida.

Tabela 4: Parâmetros dos modelos finais da Boosted Regression Trees para cada espécie de peixe. 


\section{Resumo}

O recrutamento é um processo importante no ambiente recifal, sendo o principal responsável pelo reabastecimento de populações de peixes. Esse fenômeno é altamente complexo, não sendo claro se é mais influenciado por processos estocásticos ou por processos determinísticos. No presente estudo avaliamos a dinâmica temporal do recrutamento de diversas espécies de peixes recifais, identificando sítios de berçário (i.e. recrutamento estável e alto) e a influência de variáveis ambientais. Para tal, utilizamos dados de um monitoramento de médio prazo (i.e. 2001 a 2014) realizado no Banco dos Abrolhos (BA-Brasil). Foram amostrados 47 sítios, sendo levantados dados sobre a comunidade de peixes, comunidade bentônica e outras variáveis ambientais. A partir desses dados, avaliamos a variação do recrutamento por sítio em dois períodos distintos (2001-2008/2006-2014) e a influência de variáveis ambientais no recrutamento, através da técnica Boosted Regression Trees. Constatamos que doze espécies de peixe apresentam recrutamento temporalmente estável em distintos sítios de amostragem. Também observamos um efeito positivo da densidade de peixes recifais coespecíficos adultos e da cobertura relativa de algas frondosas no recrutamento de diversas espécies analisadas. No geral, observamos que há certa espécie especificidade no processo de recrutamento, porém, em escalas espaciais maiores, os padrões podem estar ligados a características mais gerais, relacionadas a um grupo taxonômico mais elevado. Em relação aos sítios de berçário, um se destacou, sendo berçário de cinco diferentes espécies. Assim, recomendamos a criação de uma área marinha de proteção integral que englobe o sítio em questão. Além disso, este trabalho nos permite reforçar a teoria de que o recrutamento de 
peixes recifais pode ser influenciado por fenômenos determinísticos e não varia simplesmente de maneira estocástica.

Palavras-chave: Área marinha protegida, recruta, recife de coral, área de berçário, Boosted Regression Trees 


\section{Abstract}

Recruitment is an important process in the reef environment, because it is the main source of population replenishment. Reef fish recruitment is a highly complex process and it is not clear whether it is more influenced by stochastic processes or by deterministic processes. Herein, we aimed to investigate temporal dynamics of reef fish recruitment, identify nursery sites (i.e. predictably high recruitment sites) and evaluate the influence of environmental variables on recruitment. We used data from a medium-term time series (i.e. 2001-2014) of scientific surveys in Abrolhos Bank (BA-Brazil). We sampled 47 sites, for several consecutive years and recorded data about fish community, benthic community and other environmental variables. We assessed the variation of recruitment on each site, during two distinct periods (2001-2008 / 2006-2014), and used the Boosted Regression Trees technique to evaluate the influence of environmental variables in recruitment. We found that twelve reef fish species present a low temporal variable recruitment at different sampling sites. BRT showed a positive effect of the coverage of flesh algae and abundance of conspecific in the abundance of recruits (i.e. young-of-year) of many species. Overall, we notice that the recruitment traits seems to be species specific, but we also found indications that in larger spatial scales, recruitment spatial and temporal patterns may be related to general characteristics among species of the higher taxa. Nursery sites varied among species and one site was a nursery to five different reef fish species. Therefore, we recommend the creation of a new no-take marine protected area that encompasses this site. Our results also indicated that reef fish recruitment may be influenced by deterministic processes and do not vary only stochastically. 
Keywords: Marine protected areas, young-of-year, coral reefs, recruitment hotspots, Boosted Regression Trees 


\section{Introdução}

A maioria das espécies de peixes recifais tem um ciclo de vida complexo, o qual envolve a produção de larvas pelágicas que se dispersam e se desenvolvem na coluna d'água antes de retornarem e assentarem em ambiente recifal apropriado (Sale et al., 2005; Carr \& Syms, 2006). No geral, o recrutamento é definido pelo número de indivíduos (i.e. recrutas) que sobrevivem por um período arbitrário após esse assentamento (Hixon \& Webster, 2002; Myers, 2002), sendo então inseridos na população local. Entretanto, o significado de recrutamento é amplo, não existindo uma definição única aceita por todos os ecólogos (Myers, 2002; Carr \& Syms, 2006). Neste artigo consideramos recrutamento como o processo de adição de recrutas com menos de um ano à população local.

O recrutamento possui extrema importância na dinâmica populacional de peixes recifais, uma vez que é o principal mecanismo de reabastecimento de populações adultas (Carr \& Syms, 2006), podendo variar de intensidade em diversas escalas espaciais e temporais (cf. Williams \& Sale, 1981; Doherty, 1987; Milicich et al., 1992; Doherty \& Fowler, 1994a, 1994b; Ault \& Johnson, 1998; Tolimieri et al., 1998; Sale et al., 2005; Carr \& Syms, 2006). Inicialmente, acreditava-se que essa variação na intensidade do recrutamento era totalmente imprevisível, resultado apenas de fenômenos estocásticos (Eckert, 1984; Sale et al., 1984). Entretanto, esse conceito perdeu força com a constatação de distribuições espaciais não aleatórias e consistentes temporalmente em diversas escalas de espaço e tempo para diferentes espécies de peixes recifais, ou seja, de padrões espaço-temporais de recrutamento (e.g. Tolimieri et al., 1998; Sale et al., 2005; DeMartini et al., 2013). Assim, considera-se que espécies que 
apresentam padrões espaço-temporais de recrutamento devem estar não só influenciadas por processos estocásticos, mas também por processos determinísticos, os quais podem dar previsibilidade ao processo de recrutamento (Ault \& Johnson, 1998; Tolimieri et al., 1998). Atualmente, é reconhecido que o recrutamento de peixes recifais pode ser influenciado por diversos processos pré e pós-assentamento, como comportamento larval, uso de hábitat, taxa de mortalidade de recrutas, etc (cf. Williams \& Sale, 1981; Doherty, 1987; Milicich et al., 1992; Doherty \& Fowler, 1994a, 1994b; Ault \& Johnson, 1998; Tolimieri et al., 1998; Carr \& Syms, 2006).

Padrões de recrutamento estão frequentemente relacionados a processos oceanográficos de maior escala, os quais têm potencial para transportar as larvas por grandes distâncias (Carr \& Syms, 2006). Em ambiente pelágico, durante sua dispersão, larvas de várias espécies de peixes recifais podem orientar-se e detectar pistas do ambiente recifal apropriado para assentamento influenciando sua dispersão através da natação (revisado em Leis, 2006; revisado em Leis et al., 2011; Soeparno et al., 2012). Consequentemente, o comportamento larval é considerado outro possível fator que influencia os padrões de recrutamento (Tolimieri et al., 1998).

Logo após o assentamento, a mortalidade é alta, sendo a taxa de sobrevivência das larvas outra possível geradora de padrões específicos de recrutamento (Ault \& Johnson, 1998). Essa taxa apresenta variação dependente da densidade e é regida principalmente pela interação entre predação e competição (Nitschke et al., 2002; Hixon \& Jones, 2005). Além disso, a seleção e o uso de micro hábitats pelos recrutas também podem influenciar o recrutamento (Ault \& Johnson, 1998; Tolimieri, 1998; Tolimieri et al., 1998), 
sendo bem documentada a alta fidelidade entre peixes recifais juvenis e micro hábitats específicos (e.g. Tolimieri, 1995, 1998; Bell \& Kramer, 2000; Holbrook et al., 2000; Schmitt \& Holbrook, 2000; Shima, 2001; Munday, 2002; Sponaugle et al., 2002). Ademais, recrutas recém-assentados podem mover-se para um micro hábitat mais apropriado, o que também pode alterar padrões espaciais de recrutamento (Caselle \& Warner, 1996). Como se vê, apesar da influência de fatores bióticos e abióticos no recrutamento de peixes recifais ser bem documentada, sua diversidade e complexidade podem dificultar bastante 0 entendimento da dinâmica do recrutamento (Carr \& Syms, 2006).

Além das características ambientais, a implantação de Áreas Marinhas Protegidas (AMPs) e sua consequente proteção das espécies contra a pesca, também pode influenciar o recrutamento de peixes recifais, através da e sua interferência desde a reprodução das espécies até em processos pósassentamento larval (revisado em Planes et al., 2000). Estudos comprovam que populações de peixes recifais em AMPs podem exportar larvas para áreas adjacentes, auxiliando no reabastecimento de peixes em outras AMPs ou áreas não protegidas e importantes para a pesca (Planes et al., 2009; Harrison et al., 2012). Logo, AMPs têm potencial para serem elementos chave na recuperação e explotação sustentável de espécies hoje sobre-explotadas pela pesca (Ward et al., 2001).

Um dos principais papéis de áreas marinhas integralmente protegidas é o reabastecimento de populações de peixes sobre-explotadas pela pesca em áreas adjacentes (Salm et al., 2000). Portanto, em AMPs, é essencial o estudo de processos responsáveis pelo reabastecimento de populações adultas, como o recrutamento. Além disso, um conhecimento mais profundo sobre a dinâmica 
do recrutamento local pode auxiliar na avaliação da efetividade das AMPs (Planes et al., 2000; Harrison et al., 2012) e na detecção de áreas com alto potencial de recrutamento (i.e. áreas de berçário) que, se protegidas, podem aumentar a efetividade da AMP (Wen et al., 2013).

No presente estudo, analisamos o recrutamento das principais espécies de peixes recifais da região do Banco dos Abrolhos, identificando padrões espaço-temporais e áreas de berçário. Além disso, investigamos a resposta do recrutamento das principais espécies de peixe recifais à distância da costa, nível de proteção contra a pesca, composição da comunidade bentônica, profundidade média, tipo de hábitat e densidade de coespecíficos e de predadores, visando identificar as variáveis ambientais mais importantes e que poderiam ser usadas para prever o potencial de recrutamento de determinadas espécies em novas áreas. Assim, visamos fornecer uma ferramenta chave para o entendimento do recrutamento no maior complexo recifal do Atlântico Sul (Leão \& Kikuchi, 2001) e, consequentemente, auxiliar no manejo de AMPs locais.

\section{Material e métodos}

\subsection{Região de Estudo}

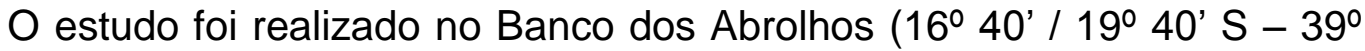
$10^{\prime} / 37^{\circ} 20^{\prime}$ W), considerado o maior e mais rico recife de coral do Atlântico Sul (Leão \& Kikuchi, 2001), sendo habitat de 265 espécies de peixes (Moura \& Francini-Filho, 2006) e 17 espécies de corais hermatípicos (Leão \& Kikuchi, 2001). O Banco dos Abrolhos engloba $42000 \mathrm{~km}^{2}$ da plataforma continental brasileira (Francini-Filho \& Moura, 2008a) e é uma região rasa, com profundidades que dificilmente excedem 30 metros (Francini-Filho et al., 2013). 
Essa região se destaca pela presença de estruturas recifais características, as quais são conhecidas como chapeirões e consistem em pináculos coralíneos em formato de cogumelo (Leão \& Kikuchi, 2001). Os chapeirões só ocorrem nessa região e podem atingir de 5 a 25 metros de altura e 20 a 300 metros de diâmetro em seu topo (Francini-Filho \& Moura, 2008a). Neste estudo do Banco dos Abrolhos foram investigadas as áreas recifais de Itacolomis, Timbebas, Arquipélago de Abrolhos, Parcel dos Abrolhos e dos Recifes Costeiros Desprotegidos, sendo que algumas estão inseridas dentro de diferentes AMPs (figura 1).

\subsection{Delineamento amostral}

No presente estudo utilizamos dados do monitoramento do Banco dos Abrolhos, realizado anualmente entre 2001 e 2014, com exceção de 2010 e 2011, durante o verão (i.e. Janeiro a Março), em diversos locais dentro de cinco diferentes áreas recifais da região. No total, amostramos 27 locais (figura 1) por diversos anos consecutivos e subdividimos cada local em 2 sítios de amostragem, um no topo do chapeirão e outro na parede (i.e. lateral do chapeirão), os quais designamos respectivamente pelos sufixos tp e pa. No caso

de dois locais de Itacolomis, não amostramos sítios de topo devido à profundidade insuficiente para permitir mergulho em segurança. Apenas os locais no Arquipélago de Abrolhos foram considerados como um sítio único cada, por serem recifes rochosos aplainados sem clara distinção entre topo e parede. Dessa maneira, na área do Arquipélago de Abrolhos, cada local corresponde a apenas um sítio, designado pelo sufixo ro. 


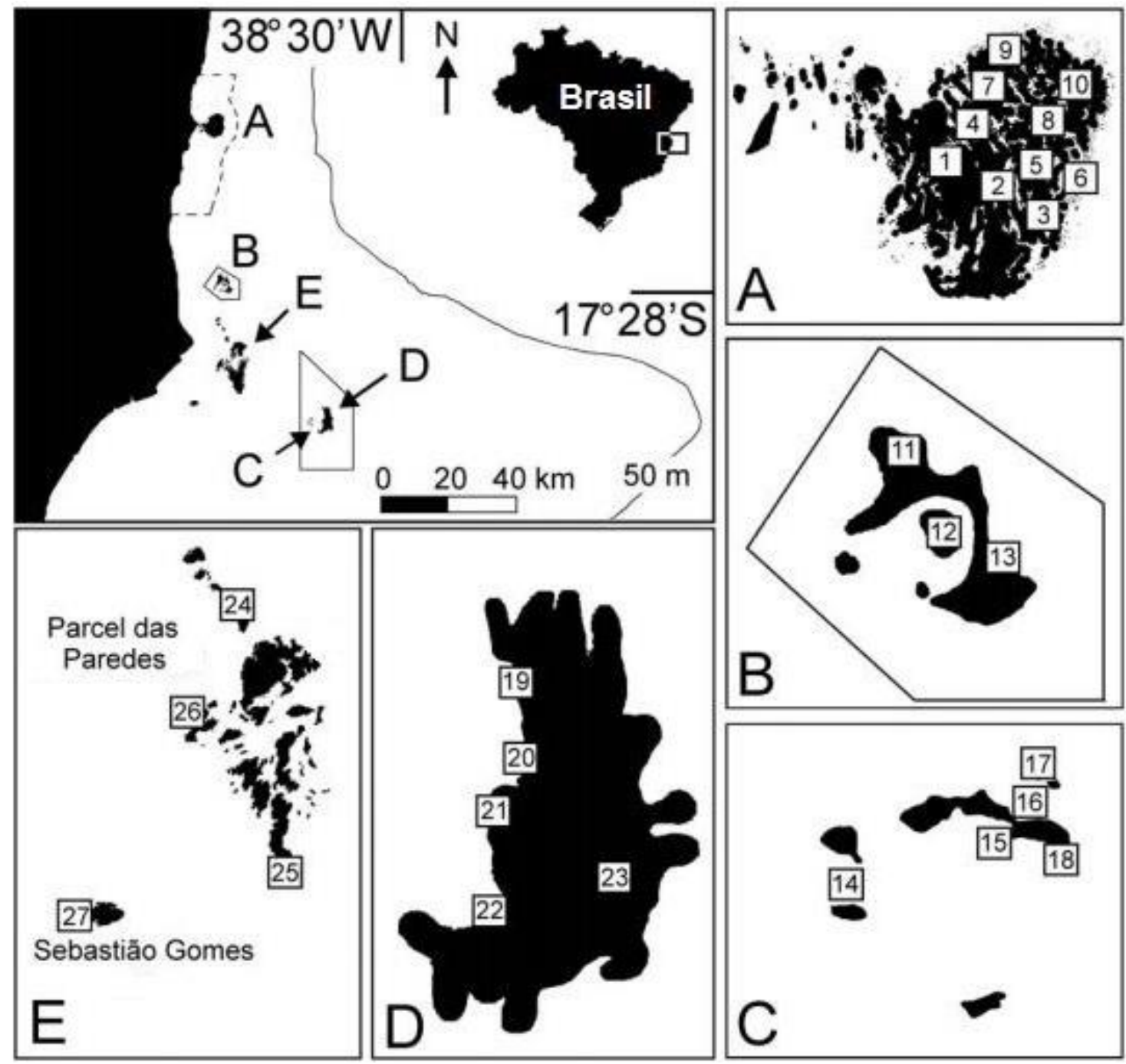

Figura 1: Croquis Topográfico do Banco dos Abrolhos - BA, mostrando suas cinco áreas recifais $A, B, C, D$ e E. Nos detalhes $A-E$, os quais estão em diferentes escalas, são representadas as posições aproximadas dos locais de amostragem por área - A) Itacolomis (Reserva Extrativista do Corumbau), B) Timbebas (Parque Nacional de Abrolhos), C) Arquipélago de Abrolhos (Parque Nacional Marinho de Abrolhos); D) Parcel dos Abrolhos (Parque Nacional de Abrolhos); E) Recifes Costeiros Desprotegidos (i.e. Sebastião Gomes e Parcel das Paredes). Para melhor caracterização dos pontos ver tabela 1.

Em cada sítio observamos a composição do bentos em 10 pontos fixos aleatoriamente dispostos (i.e. amostragem com observações repetidas ao longo da campanha), a qual foi descrita através do recobrimento percentual das espécies estimado pela técnica do fotoquadrado (quadrat) (cf. Francini-Filho et al., 2008). Nesse método cada unidade amostral é permanentemente delimitada por pinos de metal fixados no substrato e em nosso caso cobriu uma área projetada no plano do substrato de $0,7 \mathrm{~m}^{2}$, a qual foi registrada através da justaposição de 15 imagens digitais. Simultaneamente, em cada sítio, alocamos aleatoriamente outros 15 pontos correspondentes aos censos destinados a 
estimar as abundâncias das espécies de peixe (técnica do censo visual estacionário). A alocação dos censos em cada sitio foi independente entre os anos.

A técnica do censo visual estacionário (cf. Minte-Vera et al., 2008) aplicada em nosso estudo consiste na contagem de todos os indivíduos (todas as espécies de peixe) de comprimento total $(C T) \leq 10 \mathrm{~cm}$ em um raio de $2 \mathrm{~m}$ em torno do ponto marcado e, em um raio de 4 metros, dos indivíduos de CT >10 $\mathrm{cm}$, sempre com um período de 5 minutos para listagem das espécies seguido da contagem das mesmas. Além da contabilização e identificação no menor nível taxonômico possível, também foi feita a classificação de acordo com seis categorias de CT: $\leq 2$, entre $2-10,10-20,20-30,30-40$ e $>40 \mathrm{~cm}$.

Considerando todo o período de estudo, caracterizamos os sítios de acordo com sua profundidade média, distância da costa e nível de proteção, (tabela 1). Estimamos o nível de proteção contra a pesca de maneira subjetiva e atribuímos valores de 1 a 5 correspondentes a um aumento progressivo de proteção. Para isso, levamos em consideração a existência e tipo de AMP local (e.g. Parque Nacional Marinho, Reserva Extrativista, etc.), além da idade da AMP e dos recursos e eficiência na fiscalização da mesma. Assim, a classificação de se deu da seguinte maneira: 1) recife desprotegido, 2) área de uso múltiplo, 3) área de proteção integral nova 4) área de proteção integral antiga e pouco fiscalizada e 5) área de proteção integral antiga e bem fiscalizada.

Durante o monitoramento do Banco dos Abrolhos, nem todas as áreas/sítios foram amostradas por todo o período (i.e. 2001 a 2014). Por exemplo, só amostramos a área de Itacolomis até 2008 e só começamos a amostrar as áreas do Arquipélago de Abrolhos e do Parcel dos Abrolhos em 
2006. Assim, visando maximizar o número de sítios avaliados em nosso estudo, dividimos nossas análises referentes aos padrões de recrutamento e identificação de sítios de berçário em dois períodos de amostragem (a listagem dos sítios utilizados nas diferentes análise está na tabela 1).

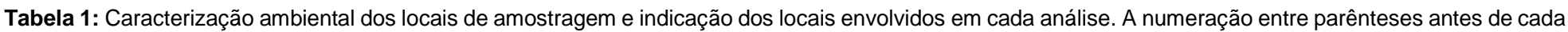
local representa sua identificação no mapa da figura 1. (TP) Topo. (PA) Parede. (RO) Rochoso.

\begin{tabular}{|c|c|c|c|c|c|c|c|}
\hline \multirow{2}{*}{\multicolumn{2}{|c|}{$\begin{array}{l}\text { Locais de amostragem nas } \\
\text { áreas }\end{array}$}} & \multirow{2}{*}{\multicolumn{2}{|c|}{$\begin{array}{l}\text { Profundidade } \\
\text { media (m) }\end{array}$}} & \multirow{2}{*}{$\begin{array}{c}\text { Distância da } \\
\text { costa }(\mathrm{km})\end{array}$} & \multirow{2}{*}{$\begin{array}{l}\text { Nível de } \\
\text { proteção }\end{array}$} & \multicolumn{2}{|c|}{$\begin{array}{c}\text { Análise padrões espaço- } \\
\text { temporais/berçários }\end{array}$} \\
\hline & & & & & & 2001-2008 & 2006-2014 \\
\hline \multicolumn{2}{|c|}{ Itacolomis: } & $\mathrm{TP}$ & PA & & & & \\
\hline & AMP1 & 1,7 & 4,6 & 5,8 & 3 & $\operatorname{Sim}$ & Não \\
\hline & AMP2 & 1,6 & 6,7 & 8,3 & 3 & $\operatorname{Sim}$ & Não \\
\hline & AMP3 & - & 11,9 & 9 & 3 & Não & Não \\
\hline (4) & $\mathrm{A} 1$ & 1,3 & 4,2 & 5,5 & 2 & Sim & Não \\
\hline (5) & A2 & 2,2 & 6,2 & 7,9 & 2 & Sim & Não \\
\hline (6) & A3 & - & 12,3 & 8,5 & 2 & Não & Não \\
\hline (7) & B1 & 1,5 & 4,5 & 5,2 & 2 & Sim & Não \\
\hline & B2 & 1,5 & 3,6 & 7,5 & 2 & $\operatorname{Sim}$ & Não \\
\hline (9) & $\mathrm{C} 1$ & 1,4 & 4,3 & 5,2 & 2 & Não & Não \\
\hline (10) & $\mathrm{C} 2$ & 2,6 & 5,6 & 7,8 & 2 & $\operatorname{Sim}$ & Não \\
\hline \multicolumn{2}{|c|}{ Timbebas: } & $\mathrm{TP}$ & PA & & & & \\
\hline (11) & TIM1 & 4,7 & 9,5 & 19,0 & 4 & Sim & $\operatorname{Sim}$ \\
\hline (12) & TIM2 & 6,0 & 11,9 & 17,4 & 4 & $\operatorname{Sim}$ & $\operatorname{Sim}$ \\
\hline (13) & TIM3 & 5,6 & 10,5 & 17,0 & 4 & $\operatorname{Sim}$ & Sim \\
\hline \multicolumn{2}{|c|}{ Parcel dos Abrolhos: } & $\mathrm{TP}$ & PA & & & & \\
\hline (23) & PAB1 & 14,7 & 5,9 & 63,4 & 5 & Não & Não \\
\hline$(21)$ & PAB2 & 7,3 & 16,9 & 61,4 & 5 & Não & Sim \\
\hline (22) & PAB3 & 6,9 & 17 & 61,7 & 5 & Não & Sim \\
\hline$(20)$ & PAB4 & 7,7 & 15,2 & 61,7 & 5 & Não & Sim \\
\hline (19) & PAB5 & 5,6 & 15 & 60,1 & 5 & Não & Sim \\
\hline \multirow{2}{*}{\multicolumn{2}{|c|}{ Recifes Costeiros Desprotegidos: }} & $\mathrm{TP}$ & PA & & & & \\
\hline & & 4,4 & 10,8 & 31,4 & 1 & $\operatorname{Sim}$ & Sim \\
\hline$(24)$ & ARENG (Arenguera) & 5,2 & 8,8 & 15,0 & 1 & Sim & Não \\
\hline$(26)$ & PLEST (Pedra de Leste) & 2,3 & 6,1 & 14,3 & 1 & $\operatorname{Sim}$ & $\operatorname{Sim}$ \\
\hline (27) & SG (Sebastião Gomes) & 3,2 & 8,2 & 13,7 & 1 & $\operatorname{Sim}$ & Sim \\
\hline \multicolumn{2}{|c|}{ Arquipélago de Abrolhos: } & RO & & & & & \\
\hline (14) & SIRIBA & 3,1 & & 58,1 & 5 & Não & Não \\
\hline (15) & MVER (Mato Verde) & 4,1 & & 58,1 & 5 & Não & Sim \\
\hline$(16)$ & PNOR (Portinho Norte) & 5,4 & & 58,1 & 5 & Não & Sim \\
\hline (17) & GUARITA & 5,4 & & 58,1 & 5 & Não & Sim \\
\hline (18) & FAROL & 5,8 & & 58,1 & 5 & Não & Sim \\
\hline
\end{tabular}

No total das campanhas (i.e. 2001 a 2014) foram realizados cerca de 7000 censos, totalizando mais de 700 horas de mergulho, nos quais foram 
visualizados e registrados mais de 40000 recrutas, pertencentes a um total de pelo menos 55 espécies de peixes recifais (anexo 1).

\subsection{Padrões espaço-temporais do recrutamento}

A partir dos dados provenientes dos censos visuais estacionários, diferenciamos os indivíduos amostrados em recrutas (i.e. indivíduos com menos de um ano) e adultos, sendo considerados recrutas os indivíduos $\leq 10 \mathrm{~cm}$ CT para espécies de grande porte (i.e. comprimento máximo $\geq 25 \mathrm{~cm} \mathrm{CT}$ ) e indivíduos $\leq 2 \mathrm{~cm}$ CT para espécies de pequeno porte (i.e. comprimento máximo $\leq 25 \mathrm{~cm} \mathrm{CT}$ ). Posteriormente, com o intuito de eliminar a interferência da variação na intensidade do recrutamento em diferentes anos de amostragem, calculamos, para cada espécie, a proporção de recrutas no sítio/ano em relação a todos os outros sítios no mesmo ano da seguinte maneira:

$$
P_{i, j, k}=\frac{\sum_{l=1}^{15} n_{i, j, k, l}}{\sum_{m=1}^{S_{k}} \sum_{l=1}^{15} n_{i, m, k, l}} \text { em que: }
$$

$\mathbf{P}_{\mathrm{i}, \mathrm{j}, \mathrm{k}}=$ Proporção de recrutas da espécie $\mathrm{i}$, no sítio $\mathrm{j}$ e no ano $\mathrm{k}$

$\mathbf{n}_{\mathrm{i}, \mathrm{j}, \mathrm{k}, \mathrm{l}}=$ Número de recrutas da espécie $\mathrm{i}$, no sítio j, no ano $\mathrm{k}$ e no censo I

$\mathbf{S}_{\mathbf{k}}=$ número total de sítios no ano $\mathrm{k}$

Feito isso, para cada sítio utilizamos o coeficiente de variação dessa proporção de recrutas ao longo dos anos como medida da variação temporal do recrutamento. Criamos um cenário nulo aleatorizando a proporção de recrutas entre os sítios dentro de cada ano de amostragem e recalculamos o coeficiente de variação por sitio ao final de cada aleatorização. Repetimos esse procedimento 10000 vezes e calculamos o valor de $p$ dividindo por 10000 o número de coeficientes de variação gerados pelas aleatorizações que foram 
iguais ou menores que o coeficiente de variação original do sítio. Consideramos um valor de $p \leq 0,05$ como indicativo de que um sítio teve um coeficiente de variação significativamente menor do que o esperado pelo acaso. Para fazer essa análise criamos uma rotina específica (anexo 2) rodada no software de domínio público R (R Core Team, 2014). Para cada espécie analisada, não consideramos os sítios que não apresentaram nenhum recruta durante o período abordado. Além disso, para cada espécie, consideramos como sendo sítios de berçário aqueles com pouca variabilidade ao longo do tempo $(p<0,05)$, e ao mesmo tempo densidades de recrutas duas vezes maior que a média calculada sem considerar sítios sem ocorrência.

Não analisamos todas as espécies observadas, pois descartamos as espécies raras ou ocasionais, que apresentaram abundância relativa de recrutas e frequência de observação menor que $10 \%$ em todos os sítios em relação a todos os recrutas de todas as espécies (a listagem espécies incluídas nas diferentes análise está na tabela 2).

Tabela 2: Listagem de espécies de peixes recifais consideradas importantes neste estudo com o tamanho dos recrutas, hábito alimentar e inclusão ou não em cada análise. $\left({ }^{*}\right)$ dados de todas as espécies do gênero Malacoctenus. $\left(^{* *}\right)$ dados das espécies Stegastes fuscus e Stegastes variabilis. ( ${ }^{* *}$ ) dados das espécies Sparisoma axillare e Sparisoma frondosum. Hábito alimentar: H, herbívoro e C, carnívoro (informações de Francini-Filho \& Moura, $\underline{2008 a) \text {. }}$

\begin{tabular}{|c|c|c|c|c|c|c|}
\hline \multirow{2}{*}{ Espécie } & \multirow{2}{*}{ Família } & \multirow{2}{*}{$\begin{array}{c}\text { Tamanho do } \\
\text { recruta do ano }(\mathrm{cm})\end{array}$} & \multirow{2}{*}{$\begin{array}{l}\text { Hábito } \\
\text { alimentar }\end{array}$} & \multicolumn{2}{|c|}{$\begin{array}{l}\text { Análise padrões espaço- } \\
\text { temporais/berçários }\end{array}$} & \multirow{2}{*}{$\begin{array}{c}\text { Análise das variáveis } \\
\text { ambientais }\end{array}$} \\
\hline & & & & 2001-2008 & 2006-2014 & \\
\hline Acanthurus bahianus & Acanthuridae & $\leq 10$ & $\mathrm{H}$ & Sim & Sim & Sim \\
\hline Acanthurus chirurgus & Acanthuridae & $\leq 10$ & $\mathrm{H}$ & Sim & Sim & Sim \\
\hline Acanthurus coeruleus & Acanthuridae & $\leq 10$ & $\mathrm{H}$ & $\operatorname{Sim}$ & Sim & $\operatorname{Sim}$ \\
\hline Anisotremus virginicus & Haemulidae & $\leq 10$ & $\mathrm{C}$ & Sim & Sim & Sim \\
\hline Haemulon aurolineatum & Haemulidae & $\leq 2$ & $\mathrm{C}$ & Sim & Não & Não \\
\hline Haemulon parra & Haemulidae & $\leq 10$ & C & $\operatorname{Sim}$ & $\operatorname{Sim}$ & Não \\
\hline Haemulon plumieri & Haemulidae & $\leq 10$ & C & Sim & $\operatorname{Sim}$ & $\operatorname{Sim}$ \\
\hline Malacoctenus spp.* & Labrisomidae & $\leq 2$ & - & Não & Não & Sim \\
\hline Ocyurus chrysurus & Lutjanidae & $\leq 10$ & C & Sim & Sim & Sim \\
\hline Abudefduf saxatilis & Pomacentridae & $\leq 2$ & - & Sim & Não & Não \\
\hline Stegastes spp.** & Pomacentridae & $\leq 2$ & - & Não & Sim & $\operatorname{Sim}$ \\
\hline Stegastes pictus & Pomacentridae & $\leq 2$ & - & Não & Sim & $\operatorname{Sim}$ \\
\hline Sparisoma spp. ${ }^{* *}$ & Labridae & $\leq 10$ & $\mathrm{H}$ & $\operatorname{Sim}$ & Sim & $\operatorname{Sim}$ \\
\hline Scarus trispnosus & Labridae & $\leq 10$ & $\mathrm{H}$ & Não & $\operatorname{Sim}$ & Não \\
\hline Scarus zellindae & Labridae & $\leq 10$ & $\mathrm{H}$ & Não & Sim & Não \\
\hline
\end{tabular}




\subsection{Resposta do recrutamento a variáveis ambientais:}

Nessa análise, avaliamos a influência da distância da costa, nível de proteção contra a pesca, profundidade, tipo de hábitat, composição da comunidade bentônica e densidade de coespecíficos e de predadores (para maiores detalhes das variáveis ambientais ver tabela 3) na densidade média de recrutas das diferentes espécies de peixe recifal por sítio e por ano. Calculamos a densidade média de recrutas através da divisão do número médio de recrutas por sítio ( $n=15$ censos) pela área amostrada por censo, ou seja, a área de um círculo de raio de $2 \mathrm{~m}$. No caso dos coespecíficos e predadores (variáveis ambientais) a área amostrada no censo foi a de um círculo de raio de $4 \mathrm{~m}$.

Utilizamos as imagens provenientes dos fotoquadrados para estimar a cobertura relativa dos diferentes tipos de organismos bentônicos por sítio. Para tal, cada imagem foi analisada no software de domínio público Coral Point Count with Excel Extentions (CPCE; Kohler \& Gill, 2006). Assim, alocamos aleatoriamente 20 pontos em cada foto e registramos qual o organismo ou, no caso de não haver nenhum, qual o substrato (rocha ou areia) exatamente abaixo de cada ponto. Para cada fotoquadrado de $0,7 \mathrm{~m}^{2}$, somaram-se os pontos por espécie nas 15 fotos dividindo-se o resultado pelo total de 300 pontos do fotoquadrado. Finalmente tirou-se a média dos 10 fotoquadrados de cada sítio (cf. Francini-Filho et al., 2008).

Para investigar a resposta do recrutamento às variáveis ambientais utilizamos a técnica Boosted Regression Trees (BRT) (Elith et al., 2008). A técnica BRT determina a melhor predição de uma variável resposta dados diversos níveis das variáveis preditoras (que podem ser qualitativas ou quantitativas), fornecendo a influência relativa de cada preditor no modelo no conjunto dos preditores. No 
presente estudo adotamos como variável resposta a densidade de recrutas por

espécie e como variáveis preditoras 28 atributos dos sítios de amostragem.

Dentre essas 28, 22 foram referentes a morfotipos entre os organismos

bentônicos e as demais 6 foram a distância da costa, o nível de proteção contra

a pesca, a profundidade, o tipo de hábitat, a densidade de coespecíficos e a densidade de predadores (tabela 3).

Tabela 3: Variáveis ambientais utilizadas como variáveis preditoras nas análises Boosted Regression Trees: códigos, descrições e unidades de medida.

\begin{tabular}{|c|c|c|}
\hline Código & Variável Ambiental & Unidade \\
\hline Hidro. ram. & Cobertura relativa do hidrocoral ramificado Millepora spp. & $\%$ \\
\hline Alga filam. & Cobertura relativa de algas filamentosas & $\%$ \\
\hline $\mathrm{ACA}$ & Cobertura relativa de algas calcárias articuladas & $\%$ \\
\hline $\mathrm{ACl}$ & Cobertura relativa de algas calcárias incrustantes & $\%$ \\
\hline Coral mas. & $\begin{array}{l}\text { Cobertura relativa dos corais hermatípicos massivos Favia spp., Mussismilia braziliensis, Mussismilia } \\
\text { hispida, Montastraea cavernosa, Meandrina braziliensis, Porites spp.e Siderastrea stellata }\end{array}$ & $\%$ \\
\hline Coral colun. & Cobertura relativa dos corais hermatípicos colunares Mussismilia hartti e Madracis decactis & $\%$ \\
\hline Coral placa & Cobertura relativa dos corais hermatípicos em forma de placa Agaricia spp. & $\%$ \\
\hline Coral incr. & Cobertura relativa do coral hermatípico incrustante Scolymia wellsi & $\%$ \\
\hline Palythoa & Cobertura relativa de Palythoa caribeorum & $\%$ \\
\hline Zoantídeo & Cobertura relativa de Zoanthus spp. & $\%$ \\
\hline Octo. ram. & $\begin{array}{l}\text { Cobertura relativa dos octocorais ramificados Carijoa riisei, Muriceopsis sulphurea, Muricia flama, } \\
\text { Neospongodes atlantica e Plexaurella spp. }\end{array}$ & $\%$ \\
\hline Octo. folha & Cobertura relativa do octocoral foliáceo Phyllogorgia dilatata & $\%$ \\
\hline Alga frond. & Cobertura relativa de algas frondosas & $\%$ \\
\hline Areia & Cobertura relativa de areia & $\%$ \\
\hline Rocha & Cobertura relativa de rocha & $\%$ \\
\hline Ouriço & Cobertura relativa de Echinometra lucunter e Lytechinus variegatus & $\%$ \\
\hline Esponja & Cobertura relativa de poríferos & $\%$ \\
\hline Ascídia & Cobertura relativa de ascídias & $\%$ \\
\hline Briozoário & Cobertura relativa de briozoários & $\%$ \\
\hline Cianobactéria & Cobertura relativa de cianobactérias & $\%$ \\
\hline Alga filam. + Ciano. & Cobertura relativa de cianobactérias associadas a algas filamentosas & $\%$ \\
\hline Outros organismos & Cobertura relativa de organismos não englobados pelas outras categorias & $\%$ \\
\hline Nível de proteção & Nível de proteção do sítio & - \\
\hline Dist. da costa & Distância do sítio até o continente & $\mathrm{km}$ \\
\hline Profundidade & Profundidade média do sítio & $\mathrm{m}$ \\
\hline Predador & $\begin{array}{l}\text { Densidade de Mycteroperca bonaci, Lutjanus jocu, Ocyurus chrysurus e Holocentrus adscensionis não- } \\
\text { recrutas (i.e. }>10 \mathrm{~cm} \text { ) }\end{array}$ & indivíduos $/ \mathrm{m}^{2}$ \\
\hline Coespecífico & Densidade de coespecíficos adultos (i.e. classes de tamanho maior que dos recrutas) & indivíduos $/ \mathrm{m}^{2}$ \\
\hline Hábitat & Identificação do tipo de hábitat: RO (rochoso), TP (topo) ou PA (parede). & - \\
\hline
\end{tabular}

Para fornecer uma medida do poder de predição do modelo, a técnica da BRT utiliza o método de validação cruzada, em que o set original de dados é 
subdividido em dois sets parciais, sendo um deles usado na construção do modelo e o outro para sua validação (Elith et al., 2008). Na técnica, é preciso definir alguns parâmetros, como o learning rate, a bag fraction e a complexidade da árvore (para maiores informações sobre os parâmetros consultar Elith et al., 2008). Assim, para cada espécie de peixe, variamos sistematicamente os parâmetros da técnica da seguinte maneira: 1) learning rate: 0,05, 0,01, 0,005 e 0,001, 2) bag fraction: 0,55, 0,65 e 0,75 e 3) complexidade da árvore: 1, 2, 3, 4 e 5. Por fim, após testarmos todas as 60 combinações possíveis desses três parâmetros, selecionamos para cada espécie a combinação que gerou o modelo de maior poder preditivo, o qual acessamos através da correlação média da validação cruzada (tabela 4). Respeitando a thumbs-rule proposta por Elith e colaboradores (Elith et al., 2008), descartamos os modelos que comportaram menos de 1000 árvores. Todos os modelos foram criados usando, no software de domínio público $\mathrm{R}$ (R Core Team, 2014), a rotina gbm.step do pacote gbm versão 2.1 (Ridgeway, 2013) combinada com o código personalizado presente no material suplementar de Elith et al., 2008.

Tabela 4: Parâmetros dos modelos finais da Boosted Regression Trees para cada espécie de peixe. (*) dados das espécies do gênero Malacoctenus. $\left(^{* *}\right)$ dados das espécies Sparisoma axillare e Sparisoma frondosum. $\left(^{* * *}\right)$ dados das espécies Stegastes fuscus e Stegastes variabilis.

\begin{tabular}{lcccc}
\multicolumn{1}{c}{ Espécie } & $\begin{array}{c}\text { Complexidade da } \\
\text { árvore }\end{array}$ & Learning Rate & Bag Fraction & Número de árvores \\
\hline Acanthurus bahianus & 3 & 0,005 & 0,75 & 1800 \\
Acanthurus chirurgus & 3 & 0,001 & 0,55 & 3700 \\
Acanthurus coeruleus & 2 & 0,001 & 0,75 & 5500 \\
Anisotremus virginicus & 1 & 0,001 & 0,75 & 1450 \\
Haemulon plumieri & 1 & 0,001 & 0,55 & 3850 \\
Malacoctenus spp. & 4 & 0,001 & 0,55 & 1550 \\
Ocyurus chrysurus & 5 & 0,001 & 0,75 & 1400 \\
Sparisoma spp. ${ }^{* *}$ & 3 & 0,005 & 0,65 & 4050 \\
Stegastes pictus & 5 & 0,001 & 0,65 & 5200 \\
Stegastes spp. ${ }^{* *}$ & 3 & 0,001 & 0,55 & 2000 \\
\hline
\end{tabular}

Nessa análise utilizamos dados de todos os sítios amostrados entre 2006 e $2008(n=138)$ e descartamos as espécies de peixe que apresentaram 
abundância relativa de recrutas e frequência de observação menor que $10 \%$ em todos os sítios no período analisado (i.e. 2006 a 2008) (as espécies analisadas pela BRT estão listadas na tabela 2). Todas as variáveis ambientais foram incluídas nas análises, porém para cada espécie de peixe apresentamos e discutimos apenas as variáveis com influência relativa $>10 \%$.

\section{Resultados}

\subsection{Padrões espaço-temporais do recrutamento}

Identificamos diversos sítios com recrutamento pouco variável temporalmente $(p<0,05)$ (figuras 2 e 3$)$. Apenas para Haemulon aurolineatum, no período de 2001 a 2008 (figura 2), e Scarus zellindae, no período de 2006 a 2014 (figura 3), não observamos sítios com recrutamento significativamente pouco variável temporalmente. As espécies variaram em relação a seus sítios de berçário, porém houve casos de 2 ou mais espécies compartilharem o(s) mesmo(s) sitio(s) de berçário, bem como de espécies com berçários específicos distintos, mas na mesma área (figuras 2 e 3).

Para o período entre 2001 a 2008 (figura 2) observamos que as áreas de Timbebas e dos Recifes Costeiros Desprotegidos apresentaram sítios de berçário para Acanthurus bahianus, Acanthurus coeruleus, Ocyurus chrysurus e Sparisoma spp. Já a região de Itacolomis apresentou sítios de berçário apenas para Haemulon plumieri e Haemulon parra. Por fim, Anisotremus virginicus, Abudefduf saxatilis e Acanthurus chirurgus, apesar de apresentarem sítios com recrutamento pouco variável temporalmente, não apresentaram sítios de berçário. 
Para o período entre 2006 e 2014 (figura 3) constatamos que a região dos Recifes Costeiros Desprotegidos e a região de Timbebas englobaram sítios de berçário de $A$. bahianus, $A$. coeruleus, Sparisoma spp. (não obrigatoriamente os mesmos de 2001-2008) e Scarus trispinosus. Já a região do Arquipélago apresentou sítios de berçário para H. plumieri, H. parra e A. bahianus. Por sua vez, a região do Parcel dos Abrolhos contemplou sítios de berçário apenas para Stegastes spp. e Stegastes pictus. Tanto A. virginicus quanto O. chrysurus, apesar de apresentarem sítios com recrutamento pouco variável, não exibiram sítios de berçário.

\subsection{Resposta do recrutamento a variáveis ambientais}

Considerando todas as espécies analisadas os modelos de BRT tiveram um poder preditivo de 0,55 , em média. Os modelos com maior poder preditivo foram os de $S$. pictus e $A$. bahianus com valores de 0,75 e 0,74 respectivamente, enquanto que os de menor poder preditivo foram os de $A$. virginicus e Stegastes spp., ambos com 0,34 (figura 4).

Em relação à influência relativa das variáveis ambientais, observamos grande variação de acordo com a espécie de peixe analisada (figura 4). Porém, para oito das dez espécies analisadas identificamos uma influência relativa maior que $10 \%$ da densidade de coespecíficos adultos. A cobertura de algas frondosas apresentou influência relativa maior que 10\% para quatro das dez espécies analisadas. Outras 12 variáveis exibiram influência relativa maior que 10\% para apenas uma espécie cada. Essas 14 variáveis foram incluídas na figura 4. 


\section{Período 2001-2008}
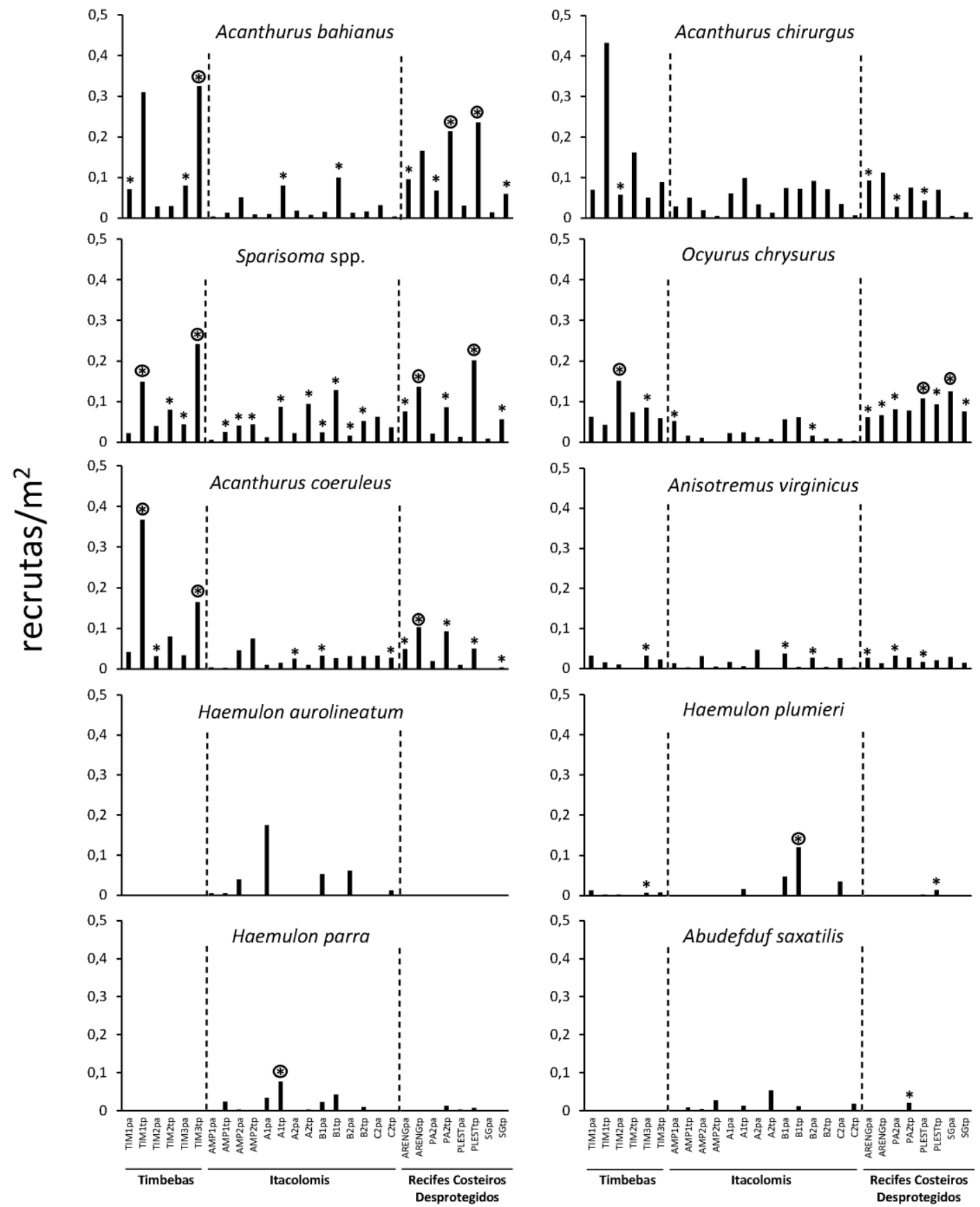

Figura 2: Recrutamento médio no período de 2001 a 2008. Densidade de recrutas (média dos anos) nos diferentes sítios de amostragem para 10 espécies de peixes recifais no Banco dos Abrolhos - BA (dados provenientes de amostragens entre 2001 e 2008). As duas últimas letras da legenda dos sítios indicam o tipo de habitat (ro=rochoso, tp=topo e pa=parede). Barras com * indicam sítios que apresentaram recrutamento com variação significativamente menor $(p<0,05)$ do que o esperado pelo acaso e aquelas que estão circuladas indicam sítios de berçário. Sparisoma spp.representa dados de recrutas das espécies Sparisoma axillare e Sparisoma frondosum. Ver designações dos sítios na tabela 1. Para facilitar a análise foram alocadas linhas tracejadas separando os sítios das diferentes áreas. 


\section{Período 2006-2014}
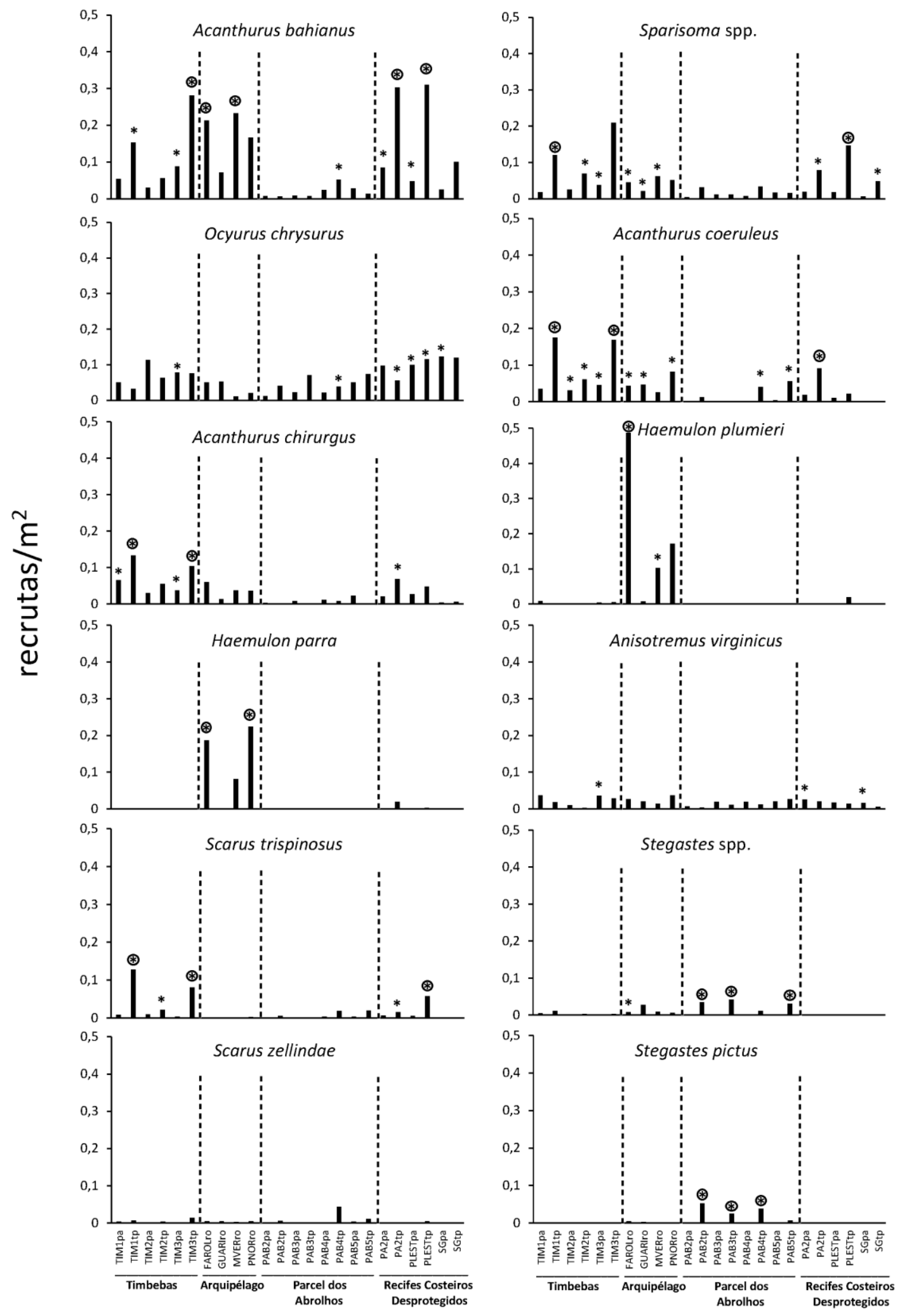

Figura 3: Recrutamento médio no período de 2006 a 2014. Densidade de recrutas (média dos anos) nos diferentes sítios de amostragem para 12 espécies de peixes recifais no Banco dos Abrolhos - BA (dados provenientes de amostragens entre 2006 e 2014). As duas últimas letras da legenda dos sítios indicam o tipo de habitat ( $r o=r o c h o s o, t p=t o p o ~ e ~ p a=p a r e d e)$. Barras com * indicam sítios que apresentaram recrutamento com variação significativamente menor $(p<0,05)$ do que o esperado pelo acaso e aquelas que estão circuladas indicam sítios de berçário. Sparisoma spp.representa dados de recrutas das espécies Sparisoma axillare e Sparisoma frondosum enquanto que Stegastes spp. representa dados de recrutas das espécies Stegastes fuscus e Stegastes variabilis. Ver designações dos sítios na tabela 1. Para facilitar a análise foram alocadas linhas tracejadas separando os sítios das diferentes áreas. 


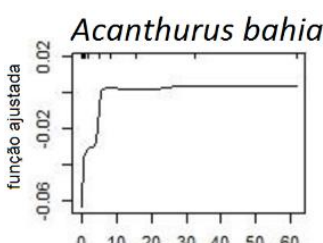

$\begin{array}{llllll}10 & 20 & 30 & 40 & 50 & 60\end{array}$

Alga frond. $(17,3 \%)$

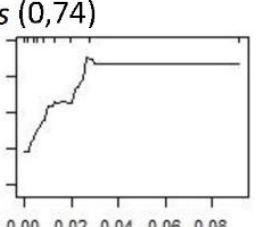

$\begin{array}{llllll}0.00 & 0.02 & 0.04 & 0.06 & 0.08\end{array}$

Coespecífico $(12,9 \%)$

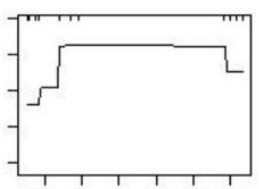

$\begin{array}{llllll}10 & 20 & 30 & 40 & 50 & 60\end{array}$

Dist. da costa $(12,5 \%)$
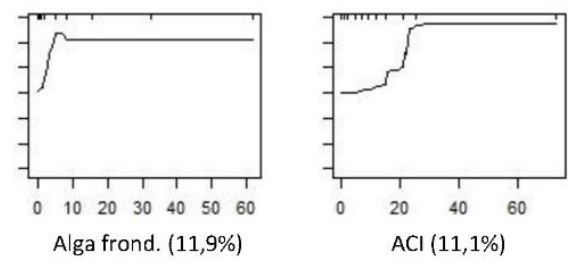

Acanthurus chirurgus $(0,40)$
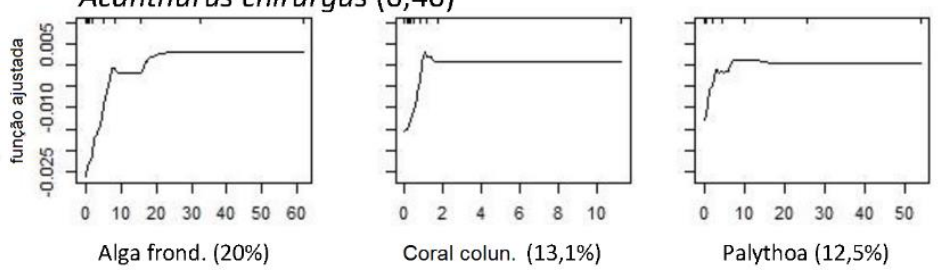

Anisotremus virginicus $(0,34)$
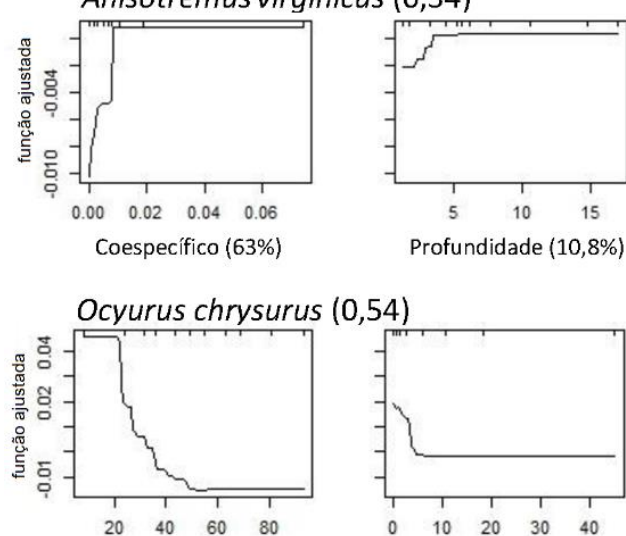

Alga filam. (30,6\%)

Alga filam. + Ciano. $(14,8 \%)$
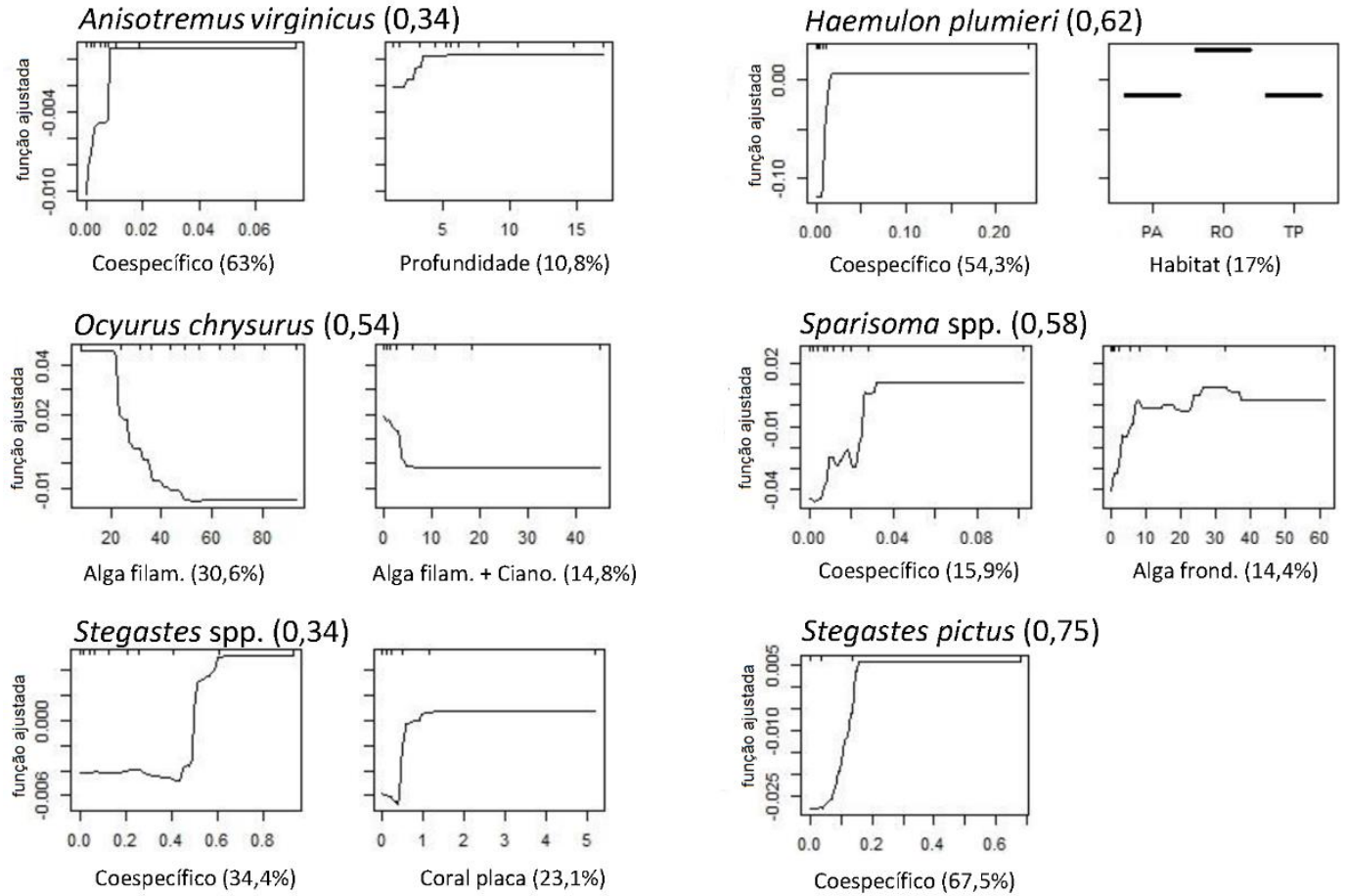

Sparisoma spp. $(0,58)$
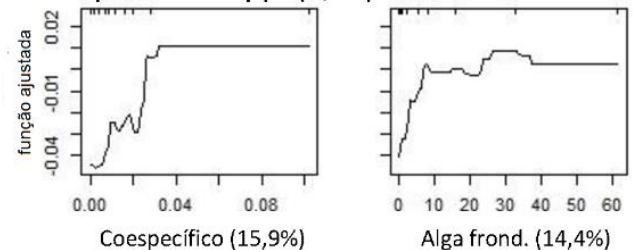

Alga frond. $(14,4 \%)$
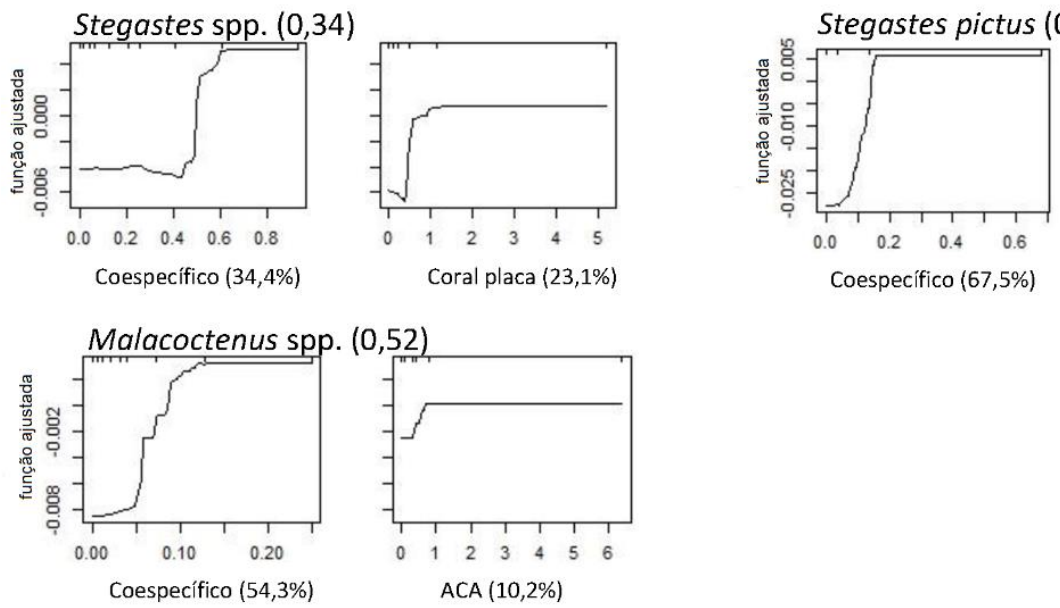

Figura 4: Gráficos de dependência parcial das variáveis ambientais mais influentes (influencia relativa > $10 \%)$ no recrutamento de 10 espécies de peixes recifais do Banco dos Abrolhos - BA. A influência relativa de cada variável ambiental é mostrada abaixo dos gráficos entre parênteses. O poder preditivo de cada modelo é mostrado, entre parênteses, ao lado do nome das espécies. Traços na borda superior interna do gráfico indicam a distribuição dos sítios de acordo com a variável ambiental. Em todos os gráficos, nas abcissas, os valores estão nas unidades originais das variáveis preditoras. Trechos ascendentes nas curvas podem ser interpretados como um crescente efeito no sentido do incremento do número de recrutas. Consulte a tabela 3 para unidades e descrição das variáveis preditoras. 


\section{Discussão}

\subsection{Padrões espaço-temporais do recrutamento}

A identificação de sítios com recrutamento estável ao longo dos anos para diversas espécies de peixes recifais indica a existência de padrões espaçotemporais para tais espécies, ou seja, repartição espacial não aleatória dos recrutas entre os sítios e ao longo do tempo. Esses padrões nos permitem reforçar a teoria de que o recrutamento de peixes recifais pode ser influenciado por fenômenos determinísticos e não varia apenas de maneira estocástica. Esse resultado corrobora outros estudos, que também constataram a presença de padrões espaço-temporais no recrutamento de outras espécies de peixes recifais em diversas localidades (e.g. Caribe: Caselle \& Warner, 1996; Tolimieri, 1998; Tolimieri et al., 1998. Açores: Fontes et al., 2009. Havaí: DeMartini et al., 2013).

Considerando a grande importância de se identificar sítios de berçário (i.e. com recrutamento alto e estável), torna-se essencial a análise dos sítios individualmente. Este tipo de análise nos permite identificar precisamente quais sítios apresentam espécies com recrutamento estável e, com os dados de densidade de recrutas, identificar quais deles são berçários. Outros trabalhos utilizaram índices de correlação de rankings (e.g. Tolimieri et al., 1998, Correlação de Spearman; Fontes et al., 2009, Coeficiente de Concordância de Kendall) para avaliar a variabilidade do recrutamento. Nesses casos, mesmo com a confirmação de padrões espaciais consistentes temporalmente, se torna arriscado eleger sítios de berçário. Por exemplo, analisando o recrutamento de A. bahianus no período de 2001 a 2008 (figura 2), notamos um claro padrão espacial com 10 sítios de recrutamento estável. Entretanto, observamos que um 
dos sítios com maior densidade média de recrutas (i.e. TIM1tp) não apresenta recrutamento estável. Dessa maneira, esse sítio poderia ser erroneamente classificado como berçário caso nossa análise não abordasse os sítios individualmente.

A partir de nossos resultados, constatamos que os sítios de berçário variam de acordo com a espécie estudada, indicando que os padrões de recrutamento são espécie específicos, fato já sugerido por outros autores (e.g. Tolimieri et al., 1998). Entretanto, observamos que os sítios de berçário de espécies de uma mesma família tendem a estar localizados em uma mesma região. Dessa maneira, os sítios de berçário dos hemulídeos aqui analisados se concentraram na área de Itacolomis (figura 2) e do Arquipélago (figura 3), os dos pomacentrídeos na área do Parcel dos Abrolhos (figura 3) e os dos acanturídeos e labrídeos na área de Timbebas e dos Recifes Costeiros Desprotegidos (figuras 2 e 3). Esse resultado nos dá indícios de que, na escala das regiões, os padrões de recrutamento podem estar relacionados a características mais generalizadas entre as espécies de um grupo taxonômico mais elevado (e.g. Família), tais como hábitos alimentares, comportamento, exigências em relação ao habitat, etc. Resultado semelhante foi encontrado por Tolimieri et al. no Caribe (1998), onde, apesar dos padrões de recrutamento constatados também serem espécie específicos, ambos acanturídeos (i.e. A. baihanus e A. coeruleus) apresentaram uma maior densidade de recrutas em um mesmo sítio e todas as espécies com hábito alimentar planctívoro apresentaram maior densidade de recrutas em um outro sítio.

Apesar dos sítios de berçário terem variado em relação à espécie, alguns sítios se destacaram por serem berçário de várias espécies simultaneamente. Esse foi o caso de TIM3tp, berçário de cinco espécies, e PLESTtp e FAROLro, 
berçários de três espécies. Juntos, esses três sítios, englobam berçários de $\mathrm{A}$. bahianus, A. coeruleus, A. chirurgus, Sparisoma spp., S. trispinosus, H. plumieri e H. parra, espécies alvo da pesca local e que representam mais da metade da biomassa de peixes recifais de Abrolhos (Francini-Filho \& Moura, 2008a). Assim, levando em conta que o FAROLro já está situado dentro de uma AMP bem fiscalizada, sugerimos maior atenção na conservação de TIM3tp e PLESTtp, visto que a primeira está inserida dentro de uma AMP pouco fiscalizada e a segunda não apresenta qualquer nível de proteção local. Abordando uma escala espacial maior, destacam-se a região de Timbebas, que engloba sítios de berçário de seis espécies, e os Recifes Costeiros Desprotegidos, que contem sítios de berçário de cinco espécies (figuras 2 e 3 ).

Além de se destacarem por serem berçário de diversas espécies, tanto TIM3tp quanto PLESTtp são sítios de berçário do budião-azul (Scarus trispinosus) (figura 3), o maior representante (i.e. maior biomassa) dos peixes papagaio (Familia labridae/tribo Scarini) em Abrolhos (Francini-Filho \& Moura, 2008a). Peixes papagaio, no geral, possuem grande importância ecológica, já que podem de remover algas e detritos do substrato e, por meio da atividade alimentar intensa, reduzir a proliferação de algas sobre corais, processo importante na manutenção da saúde dos recifes (Bonaldo et al., 2014). Além

disso S. trispinosus é uma espécie endêmica do Brasil e ameaçada de extinção (Padovani-Ferreira et al., 2012), o que a elege como uma das espécies prioritárias para conservação na região de Abrolhos.

\subsection{Resposta do recrutamento a variáveis ambientais}

Com base na validação cruzada dos modelos e considerando que coeficientes de correlação entre observações e predições em torno de 0.7 são 
apenas moderadas, constatamos que o poder preditivo de nossos modelos de BRTs em média foi baixo (coeficiente de correlação $=0,55$ ), indicando que as variáveis ambientais avaliadas em nosso estudo não explicaram consideravelmente a variabilidade da densidade de recrutas. Porém, não podemos descartar totalmente a influência dessas variáveis, uma vez que elas podem apenas ter pouca carga sobre a resposta ou então ser importantes em outras escalas espaciais. De fato, diversos estudos constataram que características bióticas do habitat local influenciam o recrutamento apenas em escalas espaciais menores (i.e. escala de metros), não apresentando influência em escalas espaciais maiores (i.e. de sítios, tal como em nossas BRT's) (e.g. Tolimieri, 1995; Caselle \& Warner, 1996; Sale et al., 2005; DeMartini et al., 2013). Isso pode explicar o baixo poder de nossos modelos, uma vez que nossa análise compreendeu a escala espacial maior (i.e. sítios) e 22 das 28 variáveis ambientais que analisamos estavam relacionadas a cobertura relativa do substrato, que variam na escala de metros, mas que por serem tratadas em média dos 10 fotoquadrados por sítio, ficaram na escala dos sítios. Logo, seriam necessários estudos adicionais, abordando uma escala espacial mais fina, de preferência amostrando a cobertura do substrato exatamente dentro da área dos censos (acoplando perfeitamente a resposta e as preditoras censo a censo).

Assim, a baixa média geral do poder preditivo de nossos modelos de BRTs indica que as variáveis analisadas não são boas preditoras do recrutamento na escala espacial abordada. Entretanto, esse resultado não quer dizer que o recrutamento de peixes recifais não seja afetado pelo ambiente; nossa constatação da existência de sítios de berçário para diversas espécies (figuras 2 e 3) indica o contrário. Outras variáveis que não analisamos devem ser as mais influentes nessa escala espacial. Realmente nós não avaliamos a 
influência de diversas outras variáveis que, em outros trabalhos, se mostraram importantes para o recrutamento de outras espécies de peixes recifais, como o aporte larval (e.g. Grorud-Colvert \& Sponaugle, 2009), características das correntes marinhas (e.g. DeMartini et al., 2013) e nível de exposição local às ondas e ao vento (e.g. DeMartini et al., 2009), entre outros. Assim, estudos abordando a resposta do recrutamento a essas (ou outras) variáveis, poderiam nos auxiliar a entender melhor os padrões espaço-temporais que identificamos.

O fato das variáveis ambientais mais influentes (influência relativa $>10 \%$ ) variarem em função da espécie analisada (figura 4) é mais um aspecto indicando que processos determinísticos tendem a influenciar o recrutamento de peixes recifais de maneira distinta dependendo da espécie estudada. Esse resultado condiz com a variabilidade que observamos em relação aos sítios de berçário, os quais foram espécie específicos. Entretanto, apesar dessa variabilidade, a densidade de coespecíficos e a cobertura relativa de algas frondosas apresentaram uma influência acima de $10 \%$ mais generalizada entre as espécies.

A densidade de coespecíficos apresentou influência relativa maior que $10 \%$ na densidade de recrutas de 8 das 10 espécies analisadas. Em todos os casos o incremento nos valores dessa variável associou-se a um efeito positivo sobre o número de recrutas, isto é, a inclinação da curva é positiva. Resultados similares, em diferentes escalas espaciais, já foram encontrados em outros estudos, os quais constataram uma correlação positiva entre densidade de recrutas e coespecíficos (e.g. Tolimieri, 1998; Demartini et al., 2013), além do efeito negativo da ausência de adultos no recrutamento de coespecíficos (Grutter, 2012). Essa maior influência da densidade de coespecíficos na densidade de recrutas em relação a outras variáveis pode ser consequência de 
diversos fatores. Primeiramente, a presença de adultos pode contribuir na diminuição da taxa de mortalidade de recrutas, umas vez que recrutas podem se beneficiar da presença de coespecíficos para aprimorar mais rapidamente seu reconhecimento a predadores (Karplus et al., 2006; Mitchell et al., 2011). Além disso, a presença de coespecíficos poderia indicar, para as larvas, a presença de hábitats com condições favoráveis para assentamento bem sucedido. Outros autores observaram que larvas de certas espécies de peixes recifais respondem positivamente a sinais de coespecíficos (Sweatman, 1988; Lecchini et al., 2005).

Além do efeito da densidade de coespecíficos ser positivo para 8 das 10 espécies analisadas, ele também se comporta de maneira semelhante para todas as espécies (ver formato das curvas na figura 4), apresentando um aumento brusco inicial e atingindo certa estabilidade a partir de determinada densidade de coespecíficos. Isso indica que aumento de coespecíficos não representa uma influência positiva constante na densidade de recrutas. Vale ressaltar que os resultados relacionados a influência da densidade de coespecíficos e o recrutamento de Stegastes spp., Malacoctenus spp. e Sparisoma spp. devem ser avaliados com cautela, já que esses dados envolvem a junção de mais de uma espécie em cada categoria.

A única outra variável com influência relativa maior que $10 \%$ no modelo de mais de uma espécie de peixe recifal (i.e. 4 espécies) foi a cobertura relativa de algas frondosas. À medida que a cobertura relativa de algas frondosas começa a aumentar a partir do mínimo, há uma intensa variação do efeito (curva muito inclinada nessa faixa das abcissas) para os três acanturídeos e para Sparisoma spp. Apesar dessas serem as únicas espécies herbívoras dessa análise (tabela 2), nosso resultado não deve estar relacionado ao uso das algas frondosas como recurso alimentar. Inclusive, já foi constatado que os herbívoros 
adultos de Abrolhos não demonstram preferência por esse recurso alimentar (Francini-Filho et al., 2010).

Dessa maneira, a influência de algas frondosas no recrutamento deve estar relacionado ao possível uso das algas frondosas como refúgio contra predação, diminuindo as taxas de mortalidade de recrutas em sítios com maior recobrimento desse tipo de alga. Resultado semelhante foi constatado no estudo de Paddack e Sponaugle (Paddack \& Sponaugle, 2008), onde foi observada uma maior associação de juvenis de Sparisoma viride com a alga Dyctiota spp. em locais com menor cobertura de corais na Flórida. Nesse estudo, a associação com essa alga frondosa foi interpretada como uma sendo uma alternativa de refúgio para os recrutas frente à diminuição da cobertura de corais no local, diminuição prevista para a região de Abrolhos (Francini-Filho et al., 2008).

Os recifes do Brasil são caracterizados pela baixa riqueza de corais hermatípicos (i.e. 18 espécies) (Leão \& Kikuchi, 2001), quando comparados a outras áreas tropicais (Roberts et al., 2002), e por não possuírem corais ramificados, que são abundantes em outras regiões (Leão \& Kikuchi, 2001) e comumente utilizados como refúgio por recrutas de peixes recifais (cf. Tolimieri, 1998; DeMartini \& Anderson, 2007; Wen et al., 2012). Assim, devido à ausência desse tipo de coral em Abrolhos, é possível que as algas frondosas assumam maior papel funcional de refúgios contra predação. Inclusive, algas frondosas já se mostraram importantes no recrutamento de peixes em ambientes temperados (Carr, 1991; Levin, 1991, 1993), onde a riqueza de corais é menor. Assim, seriam necessários estudos em uma escala espacial menor para confirmar se os recrutas de fato utilizam algas frondosas como refúgio contra predação.

Assim como a densidade de coespecíficos, o efeito da cobertura relativa de algas frondosas, quando influente no recrutamento, também apresentou um 
intenso incremento inicial seguido de estabilização a partir dos cerca de $10 \%$ de cobertura relativa (figura 4). Desse modo, a influência dessa variável parece estar mais relacionada com a influência negativa do baixo recobrimento de alga frondosas do que com uma influência positiva de um grande recobrimento. Isso era esperado já que o grande recobrimento de algas frondosas pode ter diversos efeitos negativos na saúde dos recifes de coral (Bellwood et al., 2004; Hughes et al., 2007; Bruce et al., 2012).

O fato do nível de proteção não ter apresentado influência relativa $>10 \%$ para nenhuma espécie de peixe recifal indica que fatores além da pesca (proibida nas áreas protegidas) devem ser mais influentes no recrutamento. Entretanto, esse resultado isolado, não torna dispensável a proteção dos berçários. O sucesso da proteção de berçários não obrigatoriamente se mede apenas pelo aumento do número de recrutas, mas também pelo potencial quanto ao número de adultos. Ao estudar o recrutamento de duas espécies de peixes sobre-explotados pela pesca na Flórida, concluiu-se que AMPs têm maior efetividade quando englobam berçário(s) (Wen et al., 2013). Nesse estudo, foi constatado que a densidade de peixes adultos dentro de áreas de berçário protegidas contra a pesca era de duas a três vezes maior do que em áreas protegidas que não eram berçários ou áreas desprotegidas com e sem a presença de berçários. Além disso, a densidade de adultos não diferiu entre áreas protegidas que não englobavam berçários e áreas de berçário desprotegidas. Assim, a proteção de sítios de berçário atualmente desprotegidos (e.g. PLESTtp) pode resultar em um expressivo aumento na densidade de adultos no local e consequente reabastecimento de populações em áreas não protegidas através da migração de adultos (Francini-Filho \& Moura, 2008b) ou exportação larval (Planes et al., 2009; Christie et al., 2010; Harrison et al., 2012). 


\section{Conclusões}

A partir de nosso estudo confirmamos que o recrutamento de peixes recifais é influenciado por processos determinísticos e pode manter uma distribuição espacial estável ao longo do tempo. Além disso, constatamos certa espécie-especificidade tanto na repartição espacial e dinâmica ao longo do tempo, quanto na maneira e intensidade com que diferentes variáveis influenciam no recrutamento. Entretanto, encontramos indícios de que a distribuição espacial dos recrutas em outras escalas espaciais pode estar relacionada ao modo de vida das espécies. Por fim, sugerimos que variáveis além das características do habitat e nível de proteção devem apresentar maior influência no recrutamento de peixes recifais na escala espacial analisada. Assim, seriam necessários estudos em diferentes escalas espaciais e focando outras variáveis ambientais para auxiliar na previsão do recrutamento em novas áreas de estudo.

Em relação à dinâmica do recrutamento de peixes recifais no Banco dos Abrolhos, identificamos que, apesar dos padrões serem espécie específicos, os sítios TIM3tp e PLESTtp se destacaram por serem berçário de diversas espécies, inclusive do budião-azul, espécie ameaçada de extinção e com papel chave na manutenção saúde dos recifes da região. Assim, sugerimos um aumento na fiscalização da região de Timbebas, em especial de TIM3tp, e a criação de uma nova área de proteção integral que englobe PLESTtp. Dessa maneira, visamos melhorar a efetividade na conservação do Banco dos Abrolhos, contribuindo para o modelo de gestão sustentável presente na região, o qual é baseado na criação de uma Rede de AMPs composta por Unidades de Conservação de diferentes categorias (e.g. Parques Nacionais, Reservas 
Extrativistas) e proposto pela Conservation International e seus parceiros (Dutra et al., 2011). 


\section{Bibliografia}

Ault TR, Johnson CR (1998) Relationships between habitat and recruitment of three species of damselfish (Pomacentridae) at Heron Reef, Great Barrier Reef. J Exp Mar Biol Ecol 223: 145-166

Bell T, Kramer DL (2000) Territoriality and habitat use by juvenile blue tangs Acanthurus coeruleus. Environ Biol Fish 58: 401-409

Bellwood DR, Hughes TP, Folke C, Nystrom M (2004) Confronting the coral reef crisis. Nature 429: 827-833

Bonaldo RM, Hoey AS, Bellwood DR (2014) The ecosystem roles of parrotfishes on tropical reefs. Oceanogr Mar Biol 52: 81-132.

Bruce T, Meirelles PM, Paranhos R, Rezende CE, Francini-Filho RB, Coni EOC, Vasconcelos AT, Filho GA, Hatay M, Schmieder R, Edwards R, Dinsdale E, Thompson FL (2012) Abrolhos Bank reef health evaluated by means of water quality, microbial diversity, benthic cover, and fish biomass data. PLoS ONE 7 [doi:10.1371/journal.pone.0036687]

Carr MH (1991) Habitat selection and recruitment of an assemblage of temperate zone reef fishes. J Exp Mar Biol Ecol 146: 113-137

Carr M, Syms C (2006) Recruitment. In: Allen LG, Pondella DJ, Horn MH(eds) The ecology of marine fishes: California and adjacent waters. University of California Press, Berkeley, pp 411-427

Caselle JE, Warner RR (1996) Variability in recruitment of coral reef fishes: The importance of habitat at two spatial scales. Ecology 77: 2488-2504

Christie MR, Tissot BN, Albins MA, Beets JP, Jia Y, Delisse MO, Thompson SE, Hixon MA (2010) Larval connectivity in an effective network of marine protected areas. PLOS ONE 5 [doi:10.1371/journal.pone.0015715]

DeMartini EE, Anderson TW (2007) Habitat associations and aggregation of recruit fishes on Hawaiian coral reefs. Bull Mar Sci 81: 139-152 
DeMartini EE, Zgliczynski BJ, Boland RC, Friedlander AM (2009) Influences of wind-wave exposure on the distribution and density of recruit reef fishes at Kure and Pearl and Hermes Atolls, Northwestern Hawaiian Islands. Environ Biol Fishes 85: 319-332

DeMartini EE, Wren JLK, Kobayashi DR (2013) Persistent spatial patterns of recruitment in a guild of Hawaiian coral reef fishes. Mar Ecol Prog Ser 485: 165179

Doherty, PJ (1987) The replenishment of populations of coral reef fishes, recruitment surveys, and the problems of variability manifest on multiple scales. Bull Mari Sci 41: 411-422

Doherty P J, Fowler AJ (1994a) An empirical test of recruitment limitation in a coral reef fish. Science 263: 935-939

Doherty PJ, Fowler AJ (1994b) Demograplooc consequences of variable recruitment to coral-reef fish populations - a congeneric comparison of 2 damselfishes. Bulletin of Marine Science, 54: 297-313

Dutra GF, Camargo E, Santos CAP, Ceotto P (2011) Abrolhos: challenges for the conservation and sustainable development of the area that encompasses the largest marine biodiversity in the southern Atlantic. Field Actions Sci Rep (Online), The Journal of Field Actions (Special Issue 3)

Eckert GJ (1984) Annual and spatial variation in recruitment of labroid fishes among seven reefs in the Capricorn/ Bunker Groups, Great Barrier Reef. Mar Biol 78:123-1

Fontes J, Caselle JE, Afonso P, Santos RS (2009) Multi-scale recruitment patterns and effects on local population size of a temperate reef fish. J Fish Biol 75: $1271-1286$

Francini-Filho RB, Ferreira CM, Coni EOC, Moura RL, Kaufman L (2010) Foraging activity of roving herbivorous reef fish (Acanthuridae and Scaridae) in eastern Brazil: influence of resource availability and interference competition. $J$ Mar Biol Assoc Uk 90: 481-492 
Francini-Filho RB, Moura RL (2008a) Dynamics of fish assemblages on coral reefs subjected to different management regimes in the Abrolhos Bank, eastern Brazil. Aquat Conserv 1179: 1166-1179

Francini-Filho RB, Moura RL (2008b) Evidence for spillover of reef fishes from a no-take marine reserve: an evaluation using the before-after control-impact (BACl) approach. Fish Res 93: 346-356

Francini-Filho RB, Moura RL, Thompson FL, Reis RD, Kaufman L, Kikuchi RKP, Leão ZMAN (2008) Diseases leading to accelerated decline of reef corals in the largest South Atlantic reef complex (Abrolhos Bank, eastern Brazil). Mar Pollut Bull 56: 1008-1014

Francini-Filho RB, Coni EOC, Meirelles PM, Amado-Filho GM, Thompson FL, Pereira-Filho GH, Bastos AC, Abrantes DP, Ferreira CM, Gibran FZ, Guth AZ, Sumida PYG, Oliveira NL, Kaufman L, Minte-Vera CV, Moura RL (2013) Dynamics of coral reef benthic assemblages of the Abrolhos Bank, Eastern Brazil: Inferences on natural and anthropogenic drivers. PLoS ONE 8 [doi:10.1371/journal.pone.0054260]

Grorud-Colvert K, Sponaugle S (2009) Larval supply and juvenile recruitment of coral reef fishes to marine reserves and non-reserves of the upper Florida Keys, USA. Mar Biol 156: 277-288

Grutter AS (2012) Enhanced colonization success and competition associated with conspecifics in cleaner fish Labroides dimidiatus juveniles. Coral Reefs 31 : 1169-1176

Harrison HB, Williamson DH, Evans RD, Almany GR, Thorrold SR, Russ GR, Feldheim KA, Herwerden LV, Planes S, Srinivasan M, Berumen ML, Jones GP (2012) Larval export from marine reserves and the recruitment benefit for fish and fisheries. Curr Biol 22: 1023-1028

Hixon MA, Webster MS (2002) Density dependence in reef fish populations. In: Sale PF(eds) Coral reef fishes: dynamics and diversity in a complex ecosystem. Academic Press, San Diego, pp 303-325 
Hixon MA, Jones GP (2005) Competition, predation and density-dependent mortality in demersal marine fishes. Ecology 86: 2847-2859

Holbrook SJ, Forrester GE, Schmitt RJ (2000) Spatial patterns in abundance of a damselfish reflect availability of suitable habitat. Oecologia 122: 109-120

Hughes TP, Rodrigues MJ, Bellwood DR, Ceccarelli D, Hoegh-Guldberg O, McCook L, Moltschaniwskyj N, Pratchett MS, Steneck RS, Willis B (2007) Phase shifts, herbivory, and the resilience of coral reefs to climate change. Curr Biol 17: 360-365

Karplus I, Katzenstein R, Goren M (2006) Predator recognition and social facilitation of predator avoidance in coral reef fish Dascyllus marginatus juveniles. Mar Ecol Prog Ser 319: 215-223

Kohler KE, Gill SM (2006) Coral Point Count with Excel extensions (CPCe): a Visual Basic program for the determination of coral and substrate coverage using random point count methodology. Comput Geosci 32: 1259-1269

Leão ZMAN, Kikuchi RKP (2001) The Abrolhos reefs of Brazil. In: Seeliger U, Kjerfve B(eds) Coastal marine ecosystems of Latin America. Springer-Verlag, Berlim, pp 83-96

Lecchini D, Shima J, Banaigs B, Galzin (2005) Larval sensory abilities and mechanisms of habitat selection of a coral reef fish during settlement. Oecologia 143: 326-334

Leis JM (2006) Are larvae of demersal fishes plankton or nekton? Adv Mar Biol 51 [doi: 10.1016/S0065-2881(06)51002-8]

Leis JM, Siebeck U, Dixson DL (2011) How Nemo finds home: The neuroecology of dispersal and of population connectivity in larvae of marine fishes. Integr Comp Biol 51: 826-843

Levin PS (1991) Effects of microhabitat on recruitment variation in a Gulf of Maine reef fish*. Mar Ecol Prog Ser 75: 183-189

Levin PS (1993) Habitat structure, conspecific presence and spatial variation in the recruitment of a temperate reef fish. Oecologia 94: 176-185 
Elith J, Leathwick JR, Hastie T (2008) Aworking guide to boosted regression trees. J Anim Ecol 77: 802-813

Milicich MJ, Meekan MG, Doherty PJ (1992) Larval supply: a good predictor of recruitment of three species of reef fish (Pomacentridae). Mar Ecol Prog Ser 86: 153-166

Minte-Vera CV, Moura RL, Francini-Filho RB (2008) Nested sampling: an improved visual-census technique for studying reef fish assemblages. Mar Ecol Prog Ser 367: 283-293

Mitchell MD, McCormick MI, Ferrari MCO, Chivers DP (2011) Coral reef fish rapidly learn to identify multiple unknown predators upon recruitment to the reef. PLoS ONE 6 [doi:10.1371/journal.pone.0015764]

Moura RL, Francini-Filho RB (2006) Reef and shore fishes of the Abrolhos region, Brazil. In: Dutra GF, Allen GR, Werner T, McKenna AS(eds) A rapid marine biodiversity assessment of the Abrolhos Bank, Bahia, Brazil. RAP Bulletin of Biological Assessment 38. Conservation International, Washington DC, pp 40-55 Munday PL (2002) Does habitat availability determine geographical-scale abundances of coral-dwelling fishes? Coral Reefs 21: 105-116

Myers RA (2002) Recruitment: Understanding density-dependence in fish populations. In: Hart PJB, Reynolds JD (eds) Handbook of fish biology and fisheries. Blackwell Publishing, London pp 123-148

Nitschke P, Mather M, Juanes F (2002) Evidence for density-dependent mortality in recruitment of a temperate reef fish, cunner Tautogolabrus adspersus, among similar reefs in the vicinity of an anthropogenic disturbance. Mar Ecol Prog Ser 226: $165-178$

Paddack MJ, Sponaugle S (2008) Recruitment and habitat selection of newly settled Sparisoma viride to reefs with low coral cover. Mar Ecol Prog Ser 369: 205-212

Padovani-Ferreira B, Floeter S, Rocha LA, Ferreira CE, Francini-Filho RB, Moura, RL, Gaspar AL, Feitosa C (2012) Scarus trispinosus. The IUCN Red List of Threatened Species http://www.iucnredlist.org/details/190748/0 
Planes S, Galzin R, Rubies AG, Goñi R, Harmelin J (2000) Effects of marine protected esses with special reference to Mediterranean littoral ecosystems. Environ Conserv 27: 126-143

Planes S, Jones GP, Thorrold SR (2009) Larval dispersal connects fish populations in a network of marine protected areas. Proc Natl Acad Sci U.S.A 106: 5693-5697

R Development Core Team (2014) R: A Language and environment for statistical computing (Version 3.12) [Software]. R Foundation for Statistical Computing http://www.R-project.org

Ridgeway G (2013) Gbm: generalized boosted regression models. R package version $2.1 \mathrm{http}: / /$ cran.r-project.org/web/packages/gbm/

Roberts CM, McClean CJ, Veron JEN, Hawkins JP, Allen GR, McAllister DE, Mittermeier CG, Schueler FW, Spalding M, Well F, Vynne C, Werner TB (2002) Marine biodiversity hotspots and conservation priorities for tropical reefs. Science 295: 1280-1284

Sale PF, Doherty PJ, Eckert GJ, Douglas WA, Ferrell DJ (1984) Large scale spatial and temporal variation in recruitment to fish populations on coral reefs. Oecologia 64: 191-1

Sale PF, Danilowicz BS, Doherty PJ, Williams DM (2005) The relations of microhabitat to variation in recruitment of young-of-year coral reef fishes. Bull Mar Sci 76: 123-142

Salm RV, Clark JR, Siirila E(eds) (2000) Marine and coastal protected areas: A guide for planners and managers. IUCN, Washington DC, pp 1-371

Schmitt RJ, Holbrook SJ (2000) Habitat-limited recruitment of coral reef damselfish. Ecology 81: 3479-3494

Shima S (2001) Recruitment of a Coral Reef Fish : Roles of settlement, habitat, and postsettlement losses. Ecology 82: 2190-2199

Soeparno, Nakamura Y, Yamaoka K (2013) Habitat choice and recruitment of tropical fishes on temperate coasts of Japan. Environ Biol Fish 96: 1101-1109 
Sponaugle S, Cowen RK, Shanks A, Morgan SG, Leis JM, Pineda J, Boehlert GW, Kingsford MJ, Lindeman KC, Grimes C, Munro JL (2002) Predicting selfrecruitment in marine populations: biophysical correlates and mechanisms. Bull Mar Sci 70: 341-375

Sweatman HPA (1988) Field evidence that settling coral reef fish larvae detect resident fishes using dissolved chemical cues. J Exp Mar Biol Ecol 124:163-74

Tolimieri N (1995) Effects of microhabitat characteristics on the settlement and recruitment of a coral reef fish at two spatial scales. Oecologia 102: 52-63

Tolimieri N (1998) The relationship among microhabitat characteristics, recruitment and adult abundance in the stoplight parrotfish, Sparisoma viride, at three spatial scales. Bull Mar Sci 62: 253-268

Tolimieri N, Sale PF, Nemeth R, Gestring K (1998) Replenishment of populations of Caribbean reef fishes: are spatial patterns of recruitment consistent through time? J Exp Marine Biol Ecol 230: 55-71

Ward TJ, Heinemann D, Evans N(eds) (2001) The role of marine reserves as fisheries management tools: A review of concepts, evidence and international experience. Bureau of Rural Sciences, Canberra, pp 1-192

Wen CKC, Pratchett MS, Almany GR, Jones GP (2012) Patterns of recruitment and microhabitat associations for three predatory coral reef fishes on the southern Great Barrier Reef, Australia. Coral Reefs 32: 389-398

Wen CKC, Almany GR, Williamson DH, Pratchett MS, Mannering TD, Evans RD, Leis JM, Srinivasan M, Jones GP (2013) Recruitment hotspots boost the effectiveness of no-take marine reserves. Biol Conserv 166: 124-131

Williams DM, Sale PF (1981) Spatial and temporal patterns of recruitment of juvenile coral reef fishes to coral habitats within "One Tree Lagoon", Great Barrier Reef. Mar Biol 65: 245-253 


\section{Anexos}

Anexo 1: Listagem e ocorrências das espécies de peixe com recrutas observados no Banco dos Abrolhos entre 2001 e 2014. Observação nas diferentes áreas recifais: O, observado e NO, não observado. $\left({ }^{*}\right)$ dados de mais de uma espécie.

\begin{tabular}{|c|c|c|c|c|c|c|}
\hline Família/Espécie & $\begin{array}{c}\text { Tamanho do recruta } \\
\text { do ano }(\mathrm{cm})\end{array}$ & Itacolomis & $\begin{array}{l}\text { Recifes Costeiros } \\
\text { Desprotegidos }\end{array}$ & Timbebas & $\begin{array}{l}\text { Parcel dos } \\
\text { Abrolhos }\end{array}$ & Arquipélago \\
\hline \multicolumn{7}{|l|}{ Pomacentridae } \\
\hline Abudefduf saxatilis & $<2$ & 0 & 0 & 0 & 0 & 0 \\
\hline Stegastes pictus & $<2$ & $\mathrm{O}$ & 0 & $\mathrm{O}$ & 0 & 0 \\
\hline Stegastes spp.* & $<2$ & $\mathrm{O}$ & 0 & 0 & 0 & 0 \\
\hline \multicolumn{7}{|l|}{ Scaridae } \\
\hline Sparisoma amplum & $<10$ & 0 & 0 & 0 & 0 & 0 \\
\hline Sparisoma axillare & $<10$ & $\mathrm{O}$ & 0 & $\mathrm{O}$ & 0 & 0 \\
\hline Sparisoma frondosum & $<10$ & $\mathrm{O}$ & 0 & 0 & 0 & 0 \\
\hline Scarus trispinosus & $<10$ & 0 & 0 & 0 & 0 & 0 \\
\hline Scarus zelindae & $<10$ & $\mathrm{O}$ & 0 & $\mathrm{O}$ & 0 & 0 \\
\hline \multicolumn{7}{|l|}{ Acanthuridae } \\
\hline Acanthurus bahianus & $<10$ & $\mathrm{O}$ & 0 & 0 & 0 & 0 \\
\hline Acanthurus chirurgus & $<10$ & 0 & 0 & 0 & 0 & 0 \\
\hline Acanthurus coeruleus & $<10$ & $\mathrm{O}$ & 0 & 0 & 0 & 0 \\
\hline \multicolumn{7}{|l|}{ Haemulidae } \\
\hline Anisotremus moricandi & $<10$ & $\mathrm{O}$ & NO & NO & NO & NO \\
\hline Anisotremus virginicus & $<10$ & 0 & 0 & 0 & 0 & 0 \\
\hline Haemulon aurolineatum & $<2$ & $\mathrm{O}$ & 0 & 0 & 0 & 0 \\
\hline Haemulon parra & $<10$ & 0 & 0 & 0 & 0 & 0 \\
\hline Haemulon plumieri & $<10$ & $\mathrm{O}$ & 0 & $\mathrm{O}$ & O & 0 \\
\hline \multicolumn{7}{|l|}{ Sparidae } \\
\hline Calamus pennatula & $<10$ & 0 & 0 & 0 & NO & 0 \\
\hline \multicolumn{7}{|l|}{ Sciaenidae } \\
\hline Odontoscion dentex & $<10$ & $\mathrm{O}$ & 0 & $\mathrm{O}$ & NO & NO \\
\hline Pareques Acuminatus & $<2$ & 0 & 0 & 0 & NO & 0 \\
\hline \multicolumn{7}{|l|}{ Carangidae } \\
\hline Carangoides bartholomaei & $<10$ & 0 & 0 & 0 & NO & NO \\
\hline Carangoides ruber & $<10$ & 0 & 0 & 0 & NO & NO \\
\hline Caranx latus & $<10$ & 0 & 0 & NO & NO & NO \\
\hline Pseudocaranx dentex & $<10$ & $\mathrm{O}$ & 0 & 0 & 0 & 0 \\
\hline \multicolumn{7}{|l|}{ Grammatidae } \\
\hline Gramma brasiliensis & $<2$ & $\mathrm{O}$ & 0 & 0 & 0 & 0 \\
\hline \multicolumn{7}{|l|}{ Lutjanidae } \\
\hline Lutjanus analis & $<10$ & $\mathrm{O}$ & NO & NO & NO & NO \\
\hline Lutjanus jocu & $<10$ & NO & 0 & NO & NO & NO \\
\hline Lutjanus synagris & $<10$ & 0 & 0 & 0 & NO & 0 \\
\hline Ocyurus chrysurus & $<10$ & 0 & 0 & 0 & 0 & 0 \\
\hline \multicolumn{7}{|l|}{ Mullidae } \\
\hline Pseudupeneus maculatus & $<10$ & 0 & 0 & 0 & 0 & 0 \\
\hline \multicolumn{7}{|l|}{ Chaetodontidae } \\
\hline Chaetodon striatus & $<2$ & 0 & NO & 0 & 0 & 0 \\
\hline \multicolumn{7}{|l|}{ Pomacanthidae } \\
\hline Holacanthus ciliaris & $<10$ & 0 & 0 & 0 & 0 & 0 \\
\hline Pomacanthus arcuatus & $<10$ & 0 & 0 & 0 & 0 & 0 \\
\hline Pomacanthus paru & $<10$ & 0 & 0 & 0 & 0 & 0 \\
\hline \multicolumn{7}{|l|}{ Scorpaenidae } \\
\hline Scorpaena spp.* & $<10$ & 0 & NO & NO & NO & 0 \\
\hline \multicolumn{7}{|l|}{ Diodontidae } \\
\hline Diodon hystrix & $<10$ & $\mathrm{O}$ & NO & NO & NO & NO \\
\hline
\end{tabular}


Anexo 1: Continuação

\begin{tabular}{|c|c|c|c|c|c|c|}
\hline Família/Espécie & $\begin{array}{c}\text { Tamanho do recruta } \\
\text { do ano }(\mathrm{cm})\end{array}$ & Itacolomis & $\begin{array}{l}\text { Recifes Costeiros } \\
\text { Desprotegidos }\end{array}$ & Timbebas & $\begin{array}{c}\text { Parcel dos } \\
\text { Abrolhos }\end{array}$ & Arquipélago \\
\hline \multicolumn{7}{|l|}{ Ephippidae } \\
\hline Chaetodipterus faber & $<10$ & $\mathrm{O}$ & NO & NO & NO & NO \\
\hline \multicolumn{7}{|l|}{ Synodontidae } \\
\hline Synodus spp.* & $<10$ & $\mathrm{O}$ & $\mathrm{O}$ & $\mathrm{O}$ & $\mathrm{O}$ & $\mathrm{O}$ \\
\hline \multicolumn{7}{|l|}{ Labridae } \\
\hline Bodianus rufus & $<10$ & NO & $\mathrm{O}$ & NO & NO & NO \\
\hline Halichoeres brasiliensis & $<10$ & $\mathrm{O}$ & $\mathrm{O}$ & $\mathrm{O}$ & O & $\mathrm{O}$ \\
\hline Halichoeres poeyi & $<2$ & $\mathrm{O}$ & $\mathrm{O}$ & O & $\mathrm{O}$ & $\mathrm{O}$ \\
\hline \multicolumn{7}{|l|}{ Labrisomidae } \\
\hline Malacoctenus spp.* & $<2$ & $\mathrm{O}$ & $\mathrm{O}$ & $\mathrm{O}$ & $\mathrm{O}$ & $\mathrm{O}$ \\
\hline \multicolumn{7}{|l|}{ Gobiidae } \\
\hline Coryphopterus spp.* & $<2$ & $\mathrm{O}$ & $\mathrm{O}$ & $\mathrm{O}$ & $\mathrm{O}$ & $\mathrm{O}$ \\
\hline Elacatinus figaro & $<2$ & $\mathrm{O}$ & $\mathrm{O}$ & $\mathrm{O}$ & $\mathrm{O}$ & O \\
\hline \multicolumn{7}{|l|}{ Bleniidae } \\
\hline Scartella cristata & $<2$ & $\mathrm{O}$ & NO & NO & NO & NO \\
\hline Parablennius spp.* & $<2$ & $\mathrm{O}$ & $\mathrm{O}$ & O & $\mathrm{O}$ & $\mathrm{O}$ \\
\hline \multicolumn{7}{|l|}{ Balistidae } \\
\hline Balistes vetula & $<10$ & $\mathrm{O}$ & $\mathrm{O}$ & NO & NO & NO \\
\hline \multicolumn{7}{|l|}{ Monacanthidae } \\
\hline Cantherhines pullus & $<10$ & NO & $\mathrm{O}$ & NO & NO & $\mathrm{O}$ \\
\hline \multicolumn{7}{|l|}{ Tetraodontidae } \\
\hline Canthigaster figueireidoi & $<2$ & NO & NO & NO & $\mathrm{O}$ & NO \\
\hline \multicolumn{7}{|l|}{ Serranidae } \\
\hline Cephalopholis fulva & $<10$ & NO & NO & NO & NO & $\mathrm{O}$ \\
\hline Epinephelus morio & $<10$ & NO & $\mathrm{O}$ & NO & $\mathrm{O}$ & $\mathrm{O}$ \\
\hline Mycteroperca bonaci & $<10$ & $\mathrm{O}$ & $\mathrm{O}$ & O & $\mathrm{O}$ & $\mathrm{O}$ \\
\hline Serranus flaviventris & $<2$ & $\mathrm{O}$ & $\mathrm{O}$ & $\mathrm{O}$ & $\mathrm{O}$ & NO \\
\hline \multicolumn{7}{|l|}{ Holocentridae } \\
\hline Holocentrus ascensionis & $<10$ & $\mathrm{O}$ & $\mathrm{O}$ & O & $\mathrm{O}$ & $\mathrm{O}$ \\
\hline \multicolumn{7}{|l|}{ Chaenopsidae } \\
\hline Emblemariopsis signifera & $<2$ & NO & NO & $\mathrm{O}$ & NO & NO \\
\hline
\end{tabular}


Anexo 2: Rotina para uso no software R correspondente a análise estatística dos padrões temporais de recrutamento. Comandos estão destacados em negrito. (\#) explicação do(s) comando(s) acima.

Especie.X.proporcoes.sitio.por.ano <- read.csv("Planilha espécie X.csv",sep=";",as.is=T,head=T,)

\# Carregamos a 'Planilha espécie X' (formato .csv) que está no computador e salvamos em um data.frame do R chamado 'Especie.X.proporcoes.sitio.por.ano'. Nesse arquivo estão os dados de proporção de recrutas de apenas uma espécie de peixe recifal. Dentro da planilha, cada coluna corresponde a um sítio e cada linha corresponde a um ano de amostragem. Dessa maneira, cada célula irá conter o valor da proporção de recrutas naquele sítio/ano em relação a todos os outros sítios no mesmo ano de amostragem (i.e. na mesma linha). Consulte o manuscrito principal para mais detalhes sobre o cálculo dessas proporções.

Especie.X.proporcoes.sitio.por.ano<-Especie.X.proporcoes.sitio.por.ano[,colSums(Especie.X.proporcoes.sitio.por.ano)!=0] \# Agora excluímos do data.frame as colunas que correspondem a sítios sem nenhuma ocorrência de recrutas por todo o período (i.e. média da coluna é zero).

resultados<-matrix(NA, nrow=10001, ncol=ncol(Especie.X.proporcoes.sitio.por.ano))

\# Criamos uma matriz com 10001 linhas e com 0 mesmo número de colunas do nosso data.frame "Especie.X.proporcoes.sitio.por.ano".

resultados[1,]<- (apply(Especie.X.proporcoes.sitio.por.ano,2,sd)/(apply(Especie.X.proporcoes.sitio.por.ano,2,mean)))

\# Calculamos o coeficiente de variação de cada um dos sítios e alocamos os valores na primeira linha da nossa matrix "resultados".

t1<- Especie.X.proporcoes.sitio.por.ano

\# Criamos um novo data.frame chamado "t1" com os mesmos dados do data.frame "Especie.X.proporcoes.sitio.por.ano".

for(i in 2:10001)

$\{t 1[1]<,-s a m p l e(E s p e c i e . X . p r o p o r c o e s . s i t i o . p o r . a n o[1])$,

t1[2,]<-sample(Especie.X.proporcoes.sitio.por.ano[2,])

t1[3,]<-sample(Especie.X.proporcoes.sitio.por.ano[3,])

t1[4,]<-sample(Especie.X.proporcoes.sitio.por.ano[4,])

$t 1[5]<,-s a m p l e(E s p e c i e . X . p r o p o r c o e s . s i t i o . p o r . a n o[5]$,

t1[6,]<-sample(Especie.X.proporcoes.sitio.por.ano[6,])

t1[7,]<-sample(Especie.X.proporcoes.sitio.por.ano[7,])

t1[8,]<-sample(Especie.X.proporcoes.sitio.por.ano[8,]) \#Nesse caso só vai até 8 pois nossa planilha tem 8 linhas

CVs1<-(apply(t1,2,sd)/(apply(t1,2,mean)))

CVs1<-replace(CVs1,is.nan(CVs1),0)

resultados $[i]<,-C V s 1\}$

\# Fazemos as 10000 a aleatorizações dos valores dentro de cada linha e após cada aleatorização o coeficiente de variação dos pontos é recalculado e guardado em uma das linhas da matriz "resultados".

valor.p <- rep(NA,ncol(Especie.X.proporcoes.sitio.por.ano))

\# Criamos um objeto chamado "valor.de.p" com um determinado número de espaços vagos igual ao número de colunas de

"Especie.X.proporcoes.sitio.por.ano".

for(i in 1:ncol(Especie.X.proporcoes.sitio.por.ano))

\{valor.p[i] <- sum(resultados[,i] < resultados $[1, \mathrm{i}]) / 10000\}$

\# Calculamos, por sítio, o valor de $p$ dividindo por 10000 o número de coeficientes de variação gerados pelas aleatorizações que

foram iguais ou menores que o coeficiente de variação original do sítio e guardamos no objeto "valor.p".

valor.p<-as.data.frame(valor.p, row.names=(colnames(Especie.X.proporcoes.sitio.por.ano)))

\# Acoplamos ao objeto "valor.p" o nome das colunas do data.frame "Especie.X.proporcoes.sitio.por.ano" (i.e. nome dos sítios).

valor.p

\# Acessamos o objeto "valor.p" e conferimos o valor de p para cada um dos sítios individualmente. 